\title{
An iterative scheme for split monotone variational inclusion, variational inequality and fixed point problems
}

\author{
Monairah Alansari ${ }^{1}$, Mohammad Farid ${ }^{2 *}$ (D) and Rehan Ali $^{3}$
}

\section{"Correspondence:}

mohdfrd55@gmail.com

${ }^{2}$ Department of Mathematics,

Deanship of Educational Services,

Qassim University, Buraidah 51452,

Al-Qassim, Saudi Arabia

Full list of author information is

available at the end of the article

\begin{abstract}
We propose and analyze a new type iterative algorithm to find a common solution of split monotone variational inclusion, variational inequality, and fixed point problems for an infinite family of nonexpansive mappings in the framework of Hilbert spaces. Further, we show that a sequence generated by the algorithm converges strongly to common solution. Furthermore, we list some consequences of our established theorem. Finally, we provide a numerical example to demonstrate the applicability of the algorithm. We emphasize that the result accounted in manuscript unifies and extends various results in this field of study.
\end{abstract}

MSC: 47J25; 49J40; 47H09; 90C99

Keywords: Iterative method; Strong convergence; Fixed point problem; Split monotone variational inclusion problem; Nonexpansive mapping; Variational inequality problem

\section{Introduction}

Throughout the paper, let $C_{1}$ be a nonempty subset of a real Hilbert space $H_{1}$.

A mapping $S_{1}: C_{1} \rightarrow C_{1}$ is said to be nonexpansive if

$$
\left\|S_{1} x_{1}-S_{1} x_{2}\right\| \leq\left\|x_{1}-x_{2}\right\|, \quad \forall x_{1}, x_{2} \in C_{1} .
$$

Let $\operatorname{Fix}\left(S_{1}\right)$ denote the fixed point of $S_{1}$, that is, $\operatorname{Fix}\left(S_{1}\right)=\left\{x_{1} \in C_{1}: S_{1} x_{1}=x_{1}\right\}$.

The classical scalar nonlinear variational inequality problem (in brief, VIP) is: Find $x_{1} \in$ $C_{1}$ such that

$$
\left\langle D x_{1}, x_{2}-x_{1}\right\rangle \geq 0, \quad \forall x_{2} \in C_{1}
$$

where $D: C_{1} \rightarrow H_{1}$ is a nonlinear mapping. It was introduced by Hartman and Stampacchia [1].

A mapping $S: H_{1} \rightarrow H_{1}$ is said to be

(c) The Author(s) 2020, corrected publication 2023. This article is licensed under a Creative Commons Attribution 4.0 International License, which permits use, sharing, adaptation, distribution and reproduction in any medium or format, as long as you give appropriate credit to the original author(s) and the source, provide a link to the Creative Commons licence, and indicate if changes were made. The images or other third party material in this article are included in the article's Creative Commons licence, unless indicated otherwise in a credit line to the material. If material is not included in the article's Creative Commons licence and your intended use is not permitted by statutory regulation or exceeds the permitted use, you will need to obtain permission directly from the copyright holder. To view a copy of this licence, visit http://creativecommons.org/licenses/by/4.0/. 
(i) monotone, if

$$
\left\langle S x_{1}-S x_{2}, x_{1}-x_{2}\right\rangle \geq 0, \quad \forall x_{1}, x_{2} \in H_{1}
$$

(ii) $\gamma$-inverse strongly monotone (in brief, ism), if

$$
\left\langle S x_{1}-S x_{2}, x_{1}-x_{2}\right\rangle \geq \gamma\left\|S x_{1}-S x_{2}\right\|^{2}, \quad \forall x_{1}, x_{2} \in H_{1} \text { and } \gamma>0
$$

(iii) firmly nonexpansive, if

$$
\left\langle S x_{1}-S x_{2}, x_{1}-x_{2}\right\rangle \geq\left\|S x_{1}-S x_{2}\right\|^{2}, \quad \forall x_{1}, x_{2} \in H_{1}
$$

(iv) $L$-Lipschitz continuous, if

$$
\left\|S x_{1}-S x_{2}\right\| \leq L\left\|x_{1}-x_{2}\right\|, \quad \forall x_{1}, x_{2} \in H_{1} \text { and } L>0
$$

A multi-valued mapping $M_{1}: D\left(M_{1}\right) \subseteq H_{1} \rightarrow 2^{H_{1}}$ is called monotone if, for all $x_{1}, x_{2} \in$ $D\left(M_{1}\right), u_{1} \in M_{1} x_{1}$ and $u_{2} \in M_{1} x_{2}$ such that

$$
\left\langle x_{1}-x_{2}, u_{1}-u_{2}\right\rangle \geq 0
$$

And it is maximal if $G\left(M_{1}\right)$, the graph of $M_{1}$ defined as

$$
G\left(M_{1}\right)=\left\{\left(x_{1}, u_{1}\right): u_{1} \in M_{1} x_{1}\right\}
$$

is not contained properly in the graph of other. It is well known that a monotone mapping $M_{1}$ is maximal iff for $x_{1} \in D\left(M_{1}\right), u_{1} \in H_{1},\left\langle x_{1}-x_{2}, u_{1}-u_{2}\right\rangle \geq 0$ for each $\left(x_{2}, u_{2}\right) \in G\left(M_{1}\right)$ implies that $u_{1} \in M_{1} x_{1}$.

Let $M_{1}: D\left(M_{1}\right) \subseteq H_{1} \rightarrow 2^{H_{1}}$ be a multi-valued maximal monotone mapping. Then the resolvent operator $J_{\rho_{1}}^{M_{1}}: H_{1} \rightarrow D\left(M_{1}\right)$ is defined by

$$
J_{\rho_{1}}^{M_{1}} x_{1}:=\left(1+\rho_{1} M_{1}\right)^{-1}\left(x_{1}\right), \quad \forall x_{1} \in H_{1}
$$

for $\rho_{1}>0$, where $I$ stands for the identity operator on $H_{1}$. We observe that $J_{\rho_{1}}^{M_{1}}$ is singlevalued nonexpansive and firmly nonexpansive.

Moudafi [2] was first to introduce the split monotone variational inclusion problem: Find $\tilde{x} \in H_{1}$ such that

$$
0 \in f_{1}(\tilde{x})+M_{1}(\tilde{x})
$$

and

$$
\tilde{y}=B \tilde{x} \in H_{2} \quad \text { solves } 0 \in f_{2}(\tilde{y})+M_{2}(\tilde{y})
$$

where $f_{1}: H_{1} \rightarrow H_{1}, f_{2}: H_{2} \rightarrow H_{2}$ are inverse strongly monotone mappings, $B: H_{1} \rightarrow H_{2}$ is a bounded linear mapping, and $M_{1}: H_{1} \rightarrow 2^{H_{1}}, M_{2}: H_{2} \rightarrow 2^{H_{2}}$ are multi-valued maximal monotone mappings. 
The split feasibility, split zero, and split fixed point problems are included as special cases. They have been studied broadly by various authors and solve real life problems essentially in modeling of inverse problems, sensor networks in computerized tomography and radiation therapy; for details, see [3-5].

If $f_{1} \equiv 0$ and $f_{2} \equiv 0$, then we find a split null point problem (in brief, $\mathrm{S}_{\mathrm{P}} \mathrm{NPP}$ ): Find $\tilde{x} \in H_{1}$ such that

$$
0 \in M_{1}(\tilde{x})
$$

and

$$
\tilde{y}=B \tilde{x} \in H_{2} \quad \text { solves } 0 \in M_{2}(\tilde{y}) .
$$

In this paper, we consider the split monotone variational inclusion problem (in brief, $\mathrm{S}_{\mathrm{P}}$ MVIP): Find $\tilde{x} \in H_{1}$ such that

$$
0 \in M_{1}(\tilde{x}),
$$

and

$$
\tilde{y}=B \tilde{x} \in H_{2} \quad \text { solves } 0 \in f(\tilde{y})+M_{2}(\tilde{y}) .
$$

Let $\Lambda=\left\{\tilde{x} \in H_{1}: \tilde{x} \in \operatorname{Sol}(\operatorname{MVIP}(1.6))\right.$ and $\left.B \tilde{x} \in \operatorname{Sol}(\operatorname{MVIP}(1.7))\right\}$ denote the solution of $\mathrm{S}_{\mathrm{P}} \mathrm{MVIP}(1.6)-(1.7)$.

The iterative algorithm for $\mathrm{S}_{\mathrm{P}} \mathrm{MVIP}(1.2)-(1.3)$ was introduced and studied by Moudafi [2]:

$$
x_{0} \in H_{1}, x_{n+1}=P\left(x_{n}+\eta A^{*}(Q-I) A x_{n}\right) \quad \text { for } \rho>0 \text {, }
$$

where $P:=J_{\rho}^{M_{1}}\left(I-\lambda f_{1}\right), Q:=J_{\rho}^{M_{2}}\left(I-\rho f_{2}\right), A^{*}$ is the adjoint operator of $A$ and $0<\eta<\frac{1}{\varsigma}, \varsigma$ is the spectral radius of $A^{*} A$.

The convergence analysis was studied by Byrne et al. [6] of some iterative algorithm for $\mathrm{S}_{\mathrm{P}} \mathrm{NPP}(1.4)-(1.5)$. Moreover, Kazmi et al. [7] established an iterative method to find a common solution of $S_{\mathrm{P}} \mathrm{NPP}(1.4)-(1.5)$ and fixed point problem. For instance, see [8, 9].

Recently, Qin et al. [10] proposed an algorithm for an infinite family of nonexpansive mappings as follows:

$$
x_{0} \in C_{1}, \quad x_{n+1}=\mu_{n} \theta g\left(x_{n}\right)+\eta_{n} x_{n}+\left(\left(1-\eta_{n}\right) I-\mu_{n} A\right) \mathbb{W}_{n} u_{n},
$$

where $g$ is a contraction mapping on $H_{1}, A$ is a strongly positive bounded linear operator, $W_{n}$ is generated by $S_{1}, S_{2}, \ldots$ as follows:

$$
\begin{aligned}
& \mathbb{V}_{n, n+1}:=I, \\
& \mathbb{V}_{n, n}:=\lambda_{n} S_{n} \mathbb{V}_{n, n+1}+\left(1-\lambda_{n}\right) I, \\
& \mathbb{V}_{n, n-1}:=\lambda_{n-1} S_{n-1} \mathbb{V}_{n, n}+\left(1-\lambda_{n-1}\right) I,
\end{aligned}
$$




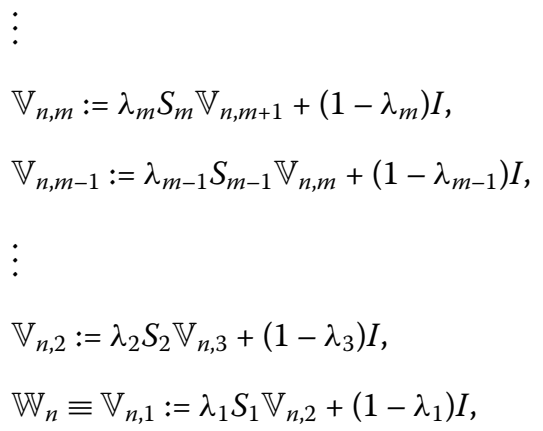

where $S_{1}, S_{2}, \ldots, W_{n}$ are nonexpansive mappings, $\left\{\lambda_{n}\right\} \subset(0,1]$ for $n \geq 1$; for further work, see $[11,12]$.

Inspirited by Moudafi [2], Byrne et al. [6], Kazmi et al. [7, 8], Qin et al. [10] and by continuing work, we propose and analyze a new type iterative algorithm to find a common solution of split monotone variational inclusion, variational inequality, and fixed point problems for an infinite family of nonexpansive mappings in the framework of Hilbert spaces. Further, we show that the sequence generated by the algorithm converges strongly to common solution. Furthermore, we list some consequences of our established theorem. Finally, we provide a numerical example to demonstrate the applicability of the algorithm. We emphasize that the result accounted in the manuscript unifies and extends various results in this field of study.

\section{Preliminaries}

This section is devoted to recalling few definitions, entailing mathematical tools, and helpful results that are required in the sequel.

To each $x_{1} \in H_{1}$, there exists a unique nearest point $P_{C_{1}} x_{1}$ to $x_{1}$ in $C_{1}$ such that

$$
\left\|x_{1}-P_{C_{1}} x_{1}\right\| \leq\left\|x_{1}-x_{2}\right\|, \quad \forall x_{2} \in C_{1},
$$

where $P_{C_{1}}$ is a metric projection of $H_{1}$ onto $C_{1}$. Also, $P_{C_{1}}$ is nonexpansive and satisfies

$$
\left\langle x_{1}-x_{2}, P_{C_{1}} x_{1}-P_{C_{1}} x_{2}\right\rangle \geq\left\|P_{C_{1}} x_{1}-P_{C_{1}} x_{2}\right\|^{2}, \quad \forall x_{1}, x_{2} \in H_{1} .
$$

Moreover, $P_{C_{1}} x_{1}$ is characterized by the fact that $P_{C_{1}} x_{1} \in C_{1}$ and

$$
\left\langle x_{1}-P_{C_{1}} x_{1}, x_{2}-P_{C_{1}} x_{1}\right\rangle \leq 0, \quad \forall x_{2} \in C_{1} .
$$

This implies that

$$
\left\|x_{1}-x_{2}\right\|^{2} \geq\left\|x_{1}-P_{C_{1}} x_{1}\right\|^{2}+\left\|x_{2}-P_{C_{1}} x_{1}\right\|^{2}, \quad \forall x_{1} \in H_{1}, x_{2} \in C_{1},
$$

and

$$
\left\|\mu x_{1}+(1-\mu) x_{2}\right\|^{2}=\mu\left\|x_{1}\right\|^{2}+(1-\mu)\left\|x_{2}\right\|^{2}-\mu(1-\mu)\left\|x_{1}-x_{2}\right\|^{2}
$$

for all $x_{1}, x_{2} \in H_{1}$ and $\mu \in[0,1]$. 
Also, on $H_{1}$ the following inequalities hold:

1. Opial's condition [13], that is, for any $\left\{x_{n}\right\}$ with $x_{n} \rightarrow x_{1}$ and

$$
\liminf _{n \rightarrow \infty}\left\|x_{n}-x_{1}\right\|<\liminf _{n \rightarrow \infty}\left\|x_{n}-x_{2}\right\|
$$

holds, $\forall x_{2} \in H_{1}$ with $x_{2} \neq x_{1}$;

2.

$$
\left\|x_{1}+x_{2}\right\|^{2} \leq\left\|x_{1}\right\|^{2}+2\left\langle x_{2}, x_{1}+x_{2}\right\rangle, \quad \forall x_{1}, x_{2} \in H_{1} .
$$

Definition 2.1 ([14]) A mapping $T_{1}: H_{1} \rightarrow H_{1}$ is called averaged iff

$$
T_{1}=(1-\lambda) I+\lambda S_{1}
$$

where $\lambda \in(0,1), I$ is the identity mapping on $H_{1}$, and $S_{1}: H_{1} \rightarrow H_{1}$ is a nonexpansive mapping.

Lemma $2.1([2])$

(i) If $T_{2}=(1-\lambda) T_{1}+\lambda S_{1}$, where $T_{1}: H_{1} \rightarrow H_{1}$ is averaged, $S_{1}: H_{1} \rightarrow H_{1}$ is nonexpansive, and $0<\lambda<1$, then $T_{2}$ is averaged;

(ii) If $T_{1}$ is $\gamma$-ism, then $\beta T_{1}$ is $\frac{\gamma}{\beta}$-ism for $\beta>0$;

(iii) $T_{1}$ is averaged iff $I-T_{1}$ is $\gamma$-ism for some $\gamma>\frac{1}{2}$.

Lemma 2.2 ([2]) Let $\rho>0, f$ be a $\gamma$-ism, and $M$ be a maximal monotone mapping. If $\rho \in(0,2 \gamma)$, then $J_{\rho}^{M}(I-\rho f)$ is averaged.

Lemma 2.3 ([2]) Let $\rho_{1}, \rho_{2}>0$ and $M_{1}, M_{2}$ be maximal monotone mappings. Then

$\tilde{x} \quad$ solves $((1.2)-(1.3)) \Leftrightarrow \quad \Leftrightarrow \quad \tilde{x}=J_{\rho_{1}}^{M_{1}}\left(I-\rho_{1} f_{1}\right) \tilde{x} \quad$ and $\quad B \tilde{x}=J_{\rho_{2}}^{M_{2}}\left(I-\rho_{2} f_{2}\right) B \tilde{x}$.

Lemma 2.4 ([15]) Let $\left\{u_{n}\right\}$ and $\left\{v_{n}\right\}$ be bounded sequences in E, a Banach space, and let $0<\mu_{n}<1$ with $0<\liminf _{n \rightarrow \infty} \mu_{n} \leq \limsup _{n \rightarrow \infty} \mu_{n}<1$. Consider $v_{n+1}=\left(1-\mu_{n}\right) v_{n}+\mu_{n} u_{n}$, $n \geq 0$ and $\lim \sup _{n \rightarrow \infty}\left(\left\|v_{n+1}-v_{n}\right\|-\left\|u_{n+1}-u_{n}\right\|\right) \leq 0$. Then

$$
\lim _{n \rightarrow \infty}\left\|v_{n}-u_{n}\right\|=0
$$

Lemma 2.5 ([16]) Assume that $B$ is a strongly positive self-adjoint bounded linear operator on $H_{1}$ with coefficient $\bar{\gamma}>0$ and $0<\rho \leq\|B\|^{-1}$. Then $\|I-\rho B\| \leq 1-\rho \bar{\gamma}$.

Lemma 2.6 ([17]) Let $\left\{a_{n}\right\}$ be a sequence of nonnegative real numbers with

$$
a_{n+1} \leq\left(1-\lambda_{n}\right) a_{n}+\alpha_{n}, \quad n \geq 0,
$$

where $\lambda_{n} \in(0,1)$ and $\left\{\alpha_{n}\right\}$ in $\mathbb{R}$ with

(i) $\sum_{n=1}^{\infty} \lambda_{n}=\infty$;

(ii) $\lim \sup _{n \rightarrow \infty} \frac{\alpha_{n}}{\lambda_{n}} \leq 0$ or $\sum_{n=1}^{\infty}\left|\alpha_{n}\right|<+\infty$.

Then $\lim _{n \rightarrow \infty} a_{n}=0$. 
Lemma 2.7 ([18]) Let $S_{1}: C_{1} \rightarrow H_{1}$ be a nonexpansive mapping. If $S_{1}$ has a fixed point, then $\left(I-S_{1}\right)$, where I is the identity mapping, it is demiclosed, that is, if $x_{n} \rightarrow x_{1} \in H_{1}$ and $x_{n}-S_{1} x_{n} \rightarrow x_{2}$, then $\left(I-S_{1}\right) x_{1}=x_{2}$.

Lemma 2.8 ([19]) Let $C_{1} \neq \emptyset$ be a closed convex subset of a strictly convex Banach space E. Let $S_{1}, S_{2}, \ldots$ be nonexpansive mappings of $C_{1}$ to $C_{1}$ such that $\bigcap_{i=1}^{\infty} \operatorname{Fix}\left(S_{i}\right) \neq \emptyset$, and let $\lambda_{1}, \lambda_{2}, \ldots$ be real numbers satisfying $0<\lambda_{i}<1, \forall i \geq 1$. Then $\lim _{i \rightarrow \infty} \mathbb{V}_{i, j} \tilde{x}$ exists, $\forall \tilde{x} \in C_{1}$ and $j \in \mathbb{N}$.

Remark 2.9 By Lemma 2.8, define a mapping $\mathbb{W}: C_{1} \rightarrow C_{1}$ such that $\mathbb{W} \tilde{x}=\lim _{i \rightarrow \infty} \mathbb{W}_{i} \tilde{x}=$ $\lim _{i \rightarrow \infty} \mathbb{V}_{i, 1} \tilde{x}, \forall \tilde{x} \in C_{1}$, which is called the $\mathbb{W}$-mapping generated by $S_{1}, S_{2}, \ldots$ and $\lambda_{1}, \lambda_{2}, \ldots$ In the whole paper, we consider $0<\lambda_{i}<1, \forall i \geq 1$.

Lemma 2.10 ([19]) Let $C_{1} \neq \emptyset$ be a closed convex subset of a strictly convex Banach space E. Let $S_{1}, S_{2}, \ldots$ be nonexpansive mappings of $C_{1}$ to $C_{1}$ such that $\bigcap_{i=1}^{\infty} \operatorname{Fix}\left(S_{i}\right) \neq \emptyset$, and let $\lambda_{1}, \lambda_{2}, \ldots$ be real numbers satisfying $0<\lambda_{i}<1, \forall i \geq 1$. Then $\operatorname{Fix}(\mathbb{W})=\bigcap_{i=1}^{\infty} \operatorname{Fix}\left(S_{i}\right)$.

Lemma 2.11 ([20]) Let $C_{1} \neq \emptyset$ be a closed convex subset of $H_{1}$. Let $S_{1}, S_{2}, \ldots$ be nonexpansive mappings of $C_{1}$ to $C_{1}$ such that $\bigcap_{i=1}^{\infty} \operatorname{Fix}\left(S_{i}\right) \neq \emptyset$, and let $\lambda_{1}, \lambda_{2}, \ldots$ be real numbers satisfying $0<\lambda_{i}<1, \forall i \geq 1$. For any bounded subset $K$ of $C_{1}, \lim _{i \rightarrow \infty} \sup _{\tilde{x} \in K}\left\|\mathbb{W}_{i} \tilde{x}-\mathbb{W} \tilde{x}\right\|=0$.

\section{Main result}

We study the following convergence result for a new type iterative method to find a common solution of $\mathrm{S}_{\mathrm{P}} \mathrm{MVIP}(1.6)-(1.7), \operatorname{VIP}(1.1)$, and fixed point problem.

Theorem 3.1 Let $H_{1}$ and $H_{2}$ denote the Hilbert spaces and $C_{1} \subset H_{1}$ be a nonempty closed convex subset of Hilbert space $H_{1}$. Let $D: C_{1} \rightarrow H_{1}$ be a $\gamma$-inverse strongly monotone mapping, $B: H_{1} \rightarrow H_{2}$ be a bounded linear operator with its adjoint operator $B^{*}, M_{1}: C_{1} \rightarrow$ $2^{H_{1}}$, and $M_{2}: H_{2} \rightarrow 2^{H_{2}}$ be multi-valued maximal monotone operators and $f: H_{2} \rightarrow H_{2}$ be an $\alpha$-inverse strongly monotone mapping. Let $g: C_{1} \rightarrow C_{1}$ be a contraction mapping with constant $\tau \in(0,1)$, A be a strongly positive bounded linear self-adjoint operator on $C_{1}$ with constant $\bar{\theta}>0$ such that $0<\theta<\frac{\bar{\theta}}{\tau}<\theta+\frac{1}{\tau}$, and $\left\{S_{i}\right\}_{i=1}^{\infty}: C_{1} \rightarrow C_{1}$ be an infinite family of nonexpansive mappings such that $\Gamma:=\Lambda \cap \operatorname{Sol}(\operatorname{VIP}(1.1)) \cap\left(\bigcap_{i=1}^{\infty} \operatorname{Fix}\left(S_{i}\right)\right) \neq \emptyset$. Let $\left\{x_{n}\right\}$ be a sequence generated as follows:

$$
\left.\begin{array}{l}
x_{1} \in C_{1}, \\
v_{n}=J_{\rho_{1}}^{M_{1}}\left[x_{n}+\eta B^{*}(Q-I) B x_{n}\right], \\
u_{n}=P_{C_{1}}\left(v_{n}-\sigma_{n} D v_{n}\right), \\
x_{n+1}=\mu_{n} \theta g\left(\mathbb{W}_{n} x_{n}\right)+\delta_{n} x_{n}+\left(\left(1-\delta_{n}\right) I-\mu_{n} A\right) \mathbb{W}_{n} u_{n}, \quad n \geq 1,
\end{array}\right\}
$$

where $\mathbb{W}_{n}$ is defined in (1.10), $Q=\int_{\rho_{2}}^{f, M_{2}}\left(I-\rho_{2} f\right),\left\{\mu_{n}\right\},\left\{\delta_{n}\right\} \subset(0,1)$ and $\eta \in\left(0, \frac{1}{\epsilon}\right), \epsilon$ is the spectral radius of $B^{*} B$. Let the control sequences satisfy the following conditions:

(i) $\lim _{n \rightarrow \infty} \mu_{n}=0, \sum_{n=1}^{\infty} \mu_{n}=\infty$;

(ii) $\rho_{1}>0,0<\rho_{2}<2 \alpha$;

(iii) $0<\liminf _{n \rightarrow \infty} \delta_{n} \leq \limsup _{n \rightarrow \infty} \delta_{n}<1$;

(iv) $0<\liminf _{n \rightarrow \infty} \sigma_{n} \leq \limsup _{n \rightarrow \infty} \sigma_{n}<2 \gamma ; \sum_{n=1}^{\infty}\left|\sigma_{n+1}-\sigma_{n}\right|<\infty$. 
Then the sequence $\left\{x_{n}\right\}$ converges strongly to some $\tilde{x} \in \Gamma$, where $\tilde{x}=P_{\Gamma}(\theta g+(I-A)) \tilde{x}$ which solves

$$
\langle(A-\theta g) \tilde{x}, v-\tilde{x}\rangle \geq 0 \quad \text { for all } v \in \Gamma .
$$

Proof For the sake of simplicity, we divide the proof into several steps.

Step 1. We prove that $\left\{x_{n}\right\}$ is bounded.

Let $\tilde{x} \in \Gamma$, then $\tilde{x} \in \Lambda$ and thus $J_{\rho_{1}}^{M_{1}} \tilde{x}=\tilde{x}, f_{\rho_{2}}^{f, M_{2}}\left(I-\rho_{2} f\right) B \tilde{x}=B \tilde{x}$ and $\left(I+\eta B^{*}(Q-I) B\right) \tilde{x}=$ $\tilde{x}$. By Lemma 2.2 and firm nonexpansiveness, $J_{\rho_{1}}^{M_{1}}$ and $f_{\rho_{2}}^{f, M_{2}}\left(I-\rho_{2} f\right)$ are averaged. Also, $\left(I+\eta B^{*}(Q-I) B\right)$ is averaged since it is $\frac{v}{\epsilon}$-ism for some $v>\frac{1}{2}$. From Lemma 2.1(iii), $I-Q$ is $v$-ism. Thus, we obtain

$$
\begin{aligned}
\left\langle B^{*}(I-Q) B x_{1}-B^{*}(I-Q) B x_{2}, x_{1}-x_{2}\right\rangle= & \left\langle(I-Q) B x_{1}-(I-Q) B x_{2},\right. \\
& \left.B x_{1}-B x_{2}\right\rangle \\
\geq & v\left\|(I-Q) B x_{1}-(I-Q) B x_{2}\right\|^{2} \\
\geq & \frac{v}{\epsilon} \| B^{*}(I-Q) B x_{1} \\
& -B^{*}(I-Q) B x_{2} \|^{2} .
\end{aligned}
$$

This implies that $\eta B^{*}(I-Q) B$ is $\frac{\nu}{\eta \epsilon}$-ism. Since $0<\eta<\frac{1}{\epsilon}$, its complement $\left(I-\eta B^{*}(I-Q) B\right)$ is averaged and hence $J_{\rho_{1}}^{M_{1}}\left[I+\eta B^{*}(Q-I) B\right]=\mathbb{R}$ (say). Thus, $I+\eta B^{*}(Q-I) B, J_{\rho_{1}}^{M_{1}}, Q$ and $\mathbb{R}$ are nonexpansive mappings.

Next, we calculate

$$
\begin{aligned}
& \left\|v_{n}-\tilde{x}\right\|^{2}=\left\|J_{\rho_{1}}^{M_{1}}\left(x_{n}+\eta B^{*}(Q-I) B x_{n}\right)-J_{\rho_{1}}^{M_{1}} \tilde{x}\right\|^{2} \\
& \leq\left\|x_{n}+\eta B^{*}(Q-I) B x_{n}-\tilde{x}\right\|^{2} \\
& =\left\|x_{n}-\tilde{x}\right\|^{2}+\eta^{2}\left\|B^{*}(Q-I) B x_{n}\right\|^{2} \\
& +2 \eta\left\langle x_{n}-\tilde{x}, B^{*}(Q-I) B x_{n}\right\rangle,
\end{aligned}
$$

and hence

$$
\begin{aligned}
\left\|v_{n}-\tilde{x}\right\|^{2} \leq & \left\|x_{n}-\tilde{x}\right\|^{2}+\eta^{2}\left\langle(Q-I) B x_{n}, B B^{*}(Q-I) B x_{n}\right\rangle \\
& +2 \eta\left\langle x_{n}-\tilde{x}, B^{*}(Q-I) B x_{n}\right\rangle .
\end{aligned}
$$

Consider $\Upsilon_{1}:=\eta^{2}\left\langle(Q-I) B x_{n}, B B^{*}(Q-I) B x_{n}\right\rangle$, and we have

$$
\begin{aligned}
\Upsilon_{1} & =\eta^{2}\left\langle(Q-I) B x_{n}, B B^{*}(Q-I) B x_{n}\right\rangle \\
& \leq \epsilon \eta^{2}\left\langle(Q-I) B x_{n},(Q-I) B x_{n}\right\rangle \\
& =\epsilon \eta^{2}\left\|(Q-I) B x_{n}\right\|^{2} .
\end{aligned}
$$

Also, let $\Upsilon_{2}:=2 \eta\left\langle x_{n}-\tilde{x}, B^{*}(Q-I) B x_{n}\right\rangle$, and we calculate

$$
\begin{aligned}
\Upsilon_{2} & =2 \eta\left\langle x_{n}-\tilde{x}, B^{*}(Q-I) B x_{n}\right\rangle \\
& =2 \eta\left\langle B\left(x_{n}-\tilde{x}\right),(Q-I) B x_{n}\right\rangle
\end{aligned}
$$




$$
\begin{aligned}
& =2 \eta\left\langle B\left(x_{n}-\tilde{x}\right)+(Q-I) B x_{n}-(Q-I) B x_{n},(Q-I) B x_{n}\right\rangle \\
& =2 \eta\left(\left\langle Q B\left(x_{n}-B \tilde{x}\right),(Q-I) B x_{n}\right\rangle-\left\|(Q-I) B x_{n}\right\|^{2}\right) \\
& \leq 2 \eta\left(\frac{1}{2}\left\|(Q-I) B x_{n}\right\|^{2}-\left\|(Q-I) B x_{n}\right\|^{2}\right. \\
& \leq-\eta\left\|(Q-I) B x_{n}\right\|^{2} .
\end{aligned}
$$

By (3.6) and (3.7) in (3.5), we get

$$
\left\|v_{n}-\tilde{x}\right\|^{2} \leq\left\|x_{n}-\tilde{x}\right\|^{2}+\eta(\epsilon \eta-1)\left\|(Q-I) B x_{n}\right\|^{2} .
$$

Since $0<\eta<\frac{1}{\epsilon}$, therefore

$$
\left\|v_{n}-\tilde{x}\right\| \leq\left\|x_{n}-\tilde{x}\right\| .
$$

Using $\gamma$-ism and $0<\sigma_{n}<2 \gamma$, we have

$$
\begin{aligned}
\left\|u_{n}-\tilde{x}\right\|^{2} & =\| P_{C_{1}}\left(v_{n}-\sigma_{n} D v_{n}\right)-P_{C_{1}}\left(v_{n}-\sigma_{n} D \tilde{x} \|^{2}\right. \\
& \leq \| v_{n}-\sigma_{n} D v_{n}-\left(v_{n}-\sigma_{n} D \tilde{x} \|^{2}\right. \\
& =\left\|\left(v_{n}-\tilde{x}\right)-\sigma_{n}\left(D v_{n}-D \tilde{x}\right)\right\|^{2} \\
& =\left\|v_{n}-\tilde{x}\right\|^{2}-2 \sigma_{n}\left\langle D v_{n}-D \tilde{x}, v_{n}-\tilde{x}\right\rangle+\sigma_{n}^{2}\left\|D v_{n}-D \tilde{x}\right\|^{2} \\
& \leq\left\|v_{n}-\tilde{x}\right\|^{2}-2 \sigma_{n} \gamma\left\|D v_{n}-D \tilde{x}\right\|^{2}+\sigma_{n}^{2}\left\|D v_{n}-D \tilde{x}\right\|^{2} \\
& =\left\|v_{n}-\tilde{x}\right\|^{2}+\sigma_{n}\left(\sigma_{n}-2 \gamma\right)\left\|D v_{n}-D \tilde{x}\right\|^{2} \\
& \leq\left\|v_{n}-\tilde{x}\right\|^{2},
\end{aligned}
$$

this implies

$$
\left\|u_{n}-\tilde{x}\right\| \leq\left\|v_{n}-\tilde{x}\right\| .
$$

By using (3.9) and (3.11), we calculate

$$
\begin{aligned}
\left\|x_{n+1}-\tilde{x}\right\|= & \left\|\mu_{n} \theta g\left(\mathbb{W}_{n} x_{n}\right)+\delta_{n} x_{n}+\left(\left(1-\delta_{n}\right) I-\mu_{n} A\right) \mathbb{W}_{n} u_{n}-\tilde{x}\right\| \\
= & \left\|\mu_{n}\left(\theta g\left(\mathbb{W}_{n} x_{n}\right)-A \tilde{x}\right)+\delta_{n}\left(x_{n}-\tilde{x}\right)+\left(\left(1-\delta_{n}\right) I-\mu_{n} A\right)\left(\mathbb{W}_{n} u_{n}-\tilde{x}\right)\right\| \\
\leq & \mu_{n}\left\|\theta g\left(\mathbb{W}_{n} x_{n}\right)-A \tilde{x}\right\|+\delta_{n}\left\|x_{n}-\tilde{x}\right\|+\left(\left(1-\delta_{n}\right) I-\mu_{n} \bar{\theta}\right)\left\|\mathbb{W}_{n} u_{n}-\tilde{x}\right\| \\
\leq & \mu_{n}\left\|\theta g\left(\mathbb{W}_{n} x_{n}\right)-\theta g(\tilde{x})+\theta g(\tilde{x})-A \tilde{x}\right\| \\
& +\delta_{n}\left\|x_{n}-\tilde{x}\right\|+\left(\left(1-\delta_{n}\right) I-\mu_{n} \bar{\theta}\right)\left\|u_{n}-\tilde{x}\right\| \\
\leq & \mu_{n} \theta\left\|g\left(\mathbb{W}_{n} x_{n}\right)-g(\tilde{x})\right\|+\mu_{n}\|\theta g(\tilde{x})-A \tilde{x}\| \\
& +\delta_{n}\left\|x_{n}-\tilde{x}\right\|+\left(\left(1-\delta_{n}\right) I-\mu_{n} \bar{\theta}\right)\left\|x_{n}-\tilde{x}\right\| \\
\leq & \mu_{n} \theta \tau\left\|x_{n}-\tilde{x}\right\|+\mu_{n}\|\theta g(\tilde{x})-A \tilde{x}\|+\left(1-\mu_{n} \bar{\theta}\right)\left\|x_{n}-\tilde{x}\right\| \\
\leq & \left(1-\mu_{n}(\bar{\theta}-\theta \tau)\right)\left\|x_{n}-\tilde{x}\right\|+\mu_{n}\|\theta g(\tilde{x})-A \tilde{x}\| \\
\leq & \max \left\{\left\|x_{n}-\tilde{x}\right\|, \frac{\|\theta g(\tilde{x})-A \tilde{x}\|}{\bar{\theta}-\theta \tau}\right\}, \quad n \geq 1 .
\end{aligned}
$$


Using induction, we get

$$
\left\|x_{n+1}-\tilde{x}\right\| \leq \max \left\{\left\|x_{1}-\tilde{x}\right\|, \frac{\|\theta g(\tilde{x})-A \tilde{x}\|}{\bar{\theta}-\theta \tau}\right\} .
$$

Thus, $\left\{x_{n}\right\}$ is bounded and hence $\left\{u_{n}\right\},\left\{\mathbb{W} u_{n}\right\}$, and $\left\{g\left(\mathbb{W}_{n} x_{n}\right)\right\}$.

Step 2. We show that $\lim _{n \rightarrow \infty}\left\|x_{n+1}-x_{n}\right\|=0, \lim _{n \rightarrow \infty}\left\|x_{n}-\mathbb{W}_{n} u_{n}\right\|=0, \lim _{n \rightarrow \infty} \| v_{n}-$ $x_{n} \|=0$, and $\lim _{n \rightarrow \infty}\left\|v_{n}-u_{n}\right\|=0$.

Since $J_{\rho_{1}}^{M_{1}}\left[I+\eta B^{*}(Q-I) B\right]$ is nonexpansive, therefore

$$
\begin{aligned}
\left\|v_{n+1}-v_{n}\right\| & =\left\|J_{\rho_{1}}^{M_{1}}\left[I+\eta B^{*}(Q-I) B\right] x_{n+1}-J_{\rho_{1}}^{M_{1}}\left[I+\eta B^{*}(Q-I) B\right] x_{n}\right\| \\
& \leq\left\|x_{n+1}-x_{n}\right\| .
\end{aligned}
$$

Using (3.13), we estimate

$$
\begin{aligned}
\left\|u_{n+1}-u_{n}\right\| & =\left\|P_{C}\left(I-\sigma_{n+1} A\right) v_{n+1}-P_{C}\left(I-\sigma_{n} S\right) v_{n}\right\| \\
& \leq\left\|\left(I-\sigma_{n+1} A\right) v_{n+1}-\left(I-\sigma_{n} A\right) v_{n}\right\| \\
& =\left\|\left(I-\sigma_{n+1} A\right) v_{n+1}-\left(I-\sigma_{n+1} A\right) v_{n}+\left(\sigma_{n}-\sigma_{n+1}\right) A v_{n}\right\| \\
& \leq\left\|v_{n+1}-v_{n}\right\|+\left|\sigma_{n}-\sigma_{n+1}\right|\left\|A v_{n}\right\| \\
& \leq\left\|x_{n+1}-x_{n}\right\|+\left|\sigma_{n}-\sigma_{n+1}\right|\left\|A v_{n}\right\| \\
& \leq\left\|x_{n+1}-x_{n}\right\|+\mathbb{N}_{1}\left|\sigma_{n}-\sigma_{n+1}\right|,
\end{aligned}
$$

where $\mathbb{N}_{1}=\sup _{n \geq 1}\left\|A v_{n}\right\|$.

For $i \in 1,2, \ldots, n, S_{i}$ and $\mathbb{V}_{n, i}$ are nonexpansive, therefore from (1.10) we obtain

$$
\begin{aligned}
\left\|\mathbb{W}_{n+1} u_{n}-\mathbb{W}_{n} u_{n}\right\| & =\left\|\lambda_{1} S_{1} \mathbb{V}_{n+1,2} u_{n}-\lambda_{1} S_{1} \mathbb{V}_{n, 2} u_{n}\right\| \\
& \leq \lambda_{1}\left\|\mathbb{V}_{n+1,2} u_{n}-\mathbb{V}_{n, 2} u_{n}\right\| \\
& \leq \lambda_{1}\left\|\lambda_{2} S_{2} \mathbb{V}_{n+1,3} u_{n}-\lambda_{2} S_{2} \mathbb{V}_{n, 3} u_{n}\right\| \\
& \leq \lambda_{1} \lambda_{2}\left\|\mathbb{V}_{n+1,3} u_{n}-\mathbb{V}_{n, 3} u_{n}\right\| \\
& \vdots \\
& \leq \lambda_{1} \lambda_{2} \ldots \lambda_{n}\left\|\mathbb{V}_{n+1, n+1} u_{n}-\mathbb{V}_{n, n+1} u_{n}\right\| \\
& \leq \mathbb{N}_{2} \prod_{i=1}^{n} \lambda_{i},
\end{aligned}
$$

where $\mathbb{N}_{2} \geq 0$ with $\left\|\mathbb{V}_{n+1, n+1} u_{n}-\mathbb{V}_{n, n+1} u_{n}\right\| \leq \mathbb{N}_{2}, \forall n \geq 1$.

Setting $x_{n+1}=\left(1-\delta_{n}\right) s_{n}+\delta_{n} x_{n}$, then we have $s_{n}=\frac{x_{n+1}-\delta_{n} x_{n}}{1-\delta_{n}}$ and

$$
\begin{aligned}
s_{n+1}-s_{n}= & \frac{\mu_{n+1} \theta g\left(\mathbb{W}_{n+1} x_{n+1}\right)+\left(\left(1-\delta_{n+1}\right) I-\mu_{n+1} A\right) \mathbb{W}_{n+1} u_{n+1}}{1-\delta_{n+1}} \\
& -\frac{\mu_{n} \theta g\left(\mathbb{W}_{n} x_{n}\right)+\left(\left(1-\delta_{n}\right) I-\mu_{n} A\right) \mathbb{W}_{n} u_{n}}{1-\delta_{n}}
\end{aligned}
$$




$$
\begin{aligned}
= & \left(\frac{\mu_{n+1}}{1-\delta_{n+1}}\right)\left(\theta g\left(\mathbb{W}_{n+1} x_{n+1}\right)-A \mathbb{W}_{n+1} u_{n+1}\right) \\
& +\left(\frac{\mu_{n}}{1-\delta_{n}}\right)\left(A \mathbb{W}_{n} u_{n}-\theta g\left(\mathbb{W}_{n} x_{n}\right)\right)+\mathbb{W}_{n+1} u_{n+1}-\mathbb{W}_{n} y_{n} \\
= & \left(\frac{\mu_{n+1}}{1-\delta_{n+1}}\right)\left(\theta g\left(\mathbb{W}_{n+1} x_{n+1}\right)-A \mathbb{W}_{n+1} u_{n+1}\right) \\
& +\left(\frac{\mu_{n}}{1-\delta_{n}}\right)\left(A \mathbb{W}_{n} u_{n}-\theta g\left(\mathbb{W}_{n} x_{n}\right)\right) \\
& +\mathbb{W}_{n+1} u_{n+1}-\mathbb{W}_{n+1} u_{n}+\mathbb{W}_{n+1} u_{n}-\mathbb{W}_{n} u_{n} .
\end{aligned}
$$

Hence,

$$
\begin{aligned}
\left\|s_{n+1}-s_{n}\right\| \leq & \frac{\mu_{n+1}}{1-\delta_{n+1}}\left(\left\|\theta g\left(\mathbb{W}_{n+1} x_{n+1}\right)\right\|+\left\|A \mathbb{W}_{n+1} u_{n+1}\right\|\right) \\
& +\frac{\mu_{n}}{1-\delta_{n}}\left(\left\|A \mathbb{W}_{n} u_{n}\right\|+\left\|\theta g\left(\mathbb{W}_{n} x_{n}\right)\right\|\right) \\
& +\left\|\mathbb{W}_{n+1} u_{n+1}-\mathbb{W}_{n+1} u_{n}\right\|+\left\|\mathbb{W}_{n+1} u_{n}-\mathbb{W}_{n} u_{n}\right\| \\
\leq & \frac{\mu_{n+1}}{1-\delta_{n+1}} \mathbb{N}_{3}+\frac{\mu_{n}}{1-\delta_{n}} \mathbb{N}_{4} \\
& +\left\|u_{n+1}-u_{n}\right\|+\left\|\mathbb{W}_{n+1} u_{n}-\mathbb{W}_{n} u_{n}\right\|
\end{aligned}
$$

where $\mathbb{N}_{3}=\sup _{n \geq 1}\left(\left\|\theta g\left(\mathbb{W}_{n+1} x_{n+1}\right)\right\|+\left\|A \mathbb{W}_{n+1} u_{n+1}\right\|\right)$ and $\mathbb{N}_{4}=\sup _{n \geq 1}\left(\left\|A \mathbb{W}_{n} u_{n}\right\|+\right.$ $\left.\left\|\theta g\left(\mathbb{W}_{n} x_{n}\right)\right\|\right)$.

Using (3.14) and (3.15) in the above inequality

$$
\begin{aligned}
\left\|s_{n+1}-s_{n}\right\| \leq & \frac{\mu_{n+1}}{1-\delta_{n+1}} \mathbb{N}_{3}+\frac{\mu_{n}}{1-\delta_{n}} \mathbb{N}_{4}+\left\|x_{n+1}-x_{n}\right\| \\
& +\mathbb{N}_{1}\left|\sigma_{n}-\sigma_{n+1}\right|+\mathbb{N}_{2} \prod_{i=1}^{n} \lambda_{i}
\end{aligned}
$$

and thus

$$
\begin{aligned}
\left\|s_{n+1}-s_{n}\right\|-\left\|x_{n+1}-x_{n}\right\| \leq & \frac{\mu_{n+1}}{1-\delta_{n+1}} \mathbb{N}_{3}+\frac{\mu_{n}}{1-\delta_{n}} \mathbb{N}_{4} \\
& +\mathbb{N}_{1}\left|\sigma_{n}-\sigma_{n+1}\right|+\mathbb{N}_{2} \prod_{i=1}^{n} \lambda_{i} .
\end{aligned}
$$

Using the given conditions in the above inequality, we have

$$
\limsup _{n \rightarrow \infty}\left(\left\|s_{n+1}-s_{n}\right\|-\left\|x_{n+1}-x_{n}\right\|\right) \leq 0
$$

By Lemma 2.4, we get

$$
\lim _{n \rightarrow \infty}\left\|s_{n}-x_{n}\right\|=0
$$

As $x_{n+1}=\left(1-\delta_{n}\right) s_{n}+\delta_{n} x_{n}$ therefore

$$
\left\|x_{n+1}-x_{n}\right\|=\left\|\left(1-\delta_{n}\right)\left(s_{n}-x_{n}\right)\right\|,
$$


which yields

$$
\lim _{n \rightarrow \infty}\left\|x_{n+1}-x_{n}\right\|=0 .
$$

Now,

$$
\begin{aligned}
\left\|x_{n}-\mathbb{W}_{n} u_{n}\right\|= & \left\|x_{n}-x_{n+1}+x_{n+1}-\mathbb{W}_{n} u_{n}\right\| \\
\leq & \left\|x_{n+1}-x_{n}\right\|+\| \mu_{n} \theta g\left(\mathbb{W}_{n} x_{n}\right)+\delta_{n} x_{n} \\
& +\left(\left(1-\delta_{n}\right) I-\mu_{n} A\right) \mathbb{W}_{n} u_{n}-\mathbb{W}_{n} u_{n} \| \\
= & \left\|x_{n+1}-x_{n}\right\|+\left\|\mu_{n}\left(\theta g\left(\mathbb{W}_{n} x_{n}\right)-A \mathbb{W}_{n} u_{n}\right)\right\| \\
& +\left(\left(1-\delta_{n}\right) I-\mu_{n} A\right)\left(\mathbb{W}_{n} u_{n}-\mathbb{W}_{n} u_{n}\right)+\delta_{n}\left(x_{n}-\mathbb{W}_{n} u_{n}\right) \\
\leq & \left\|x_{n+1}-x_{n}\right\|+\mu_{n}\left\|\theta g\left(\mathbb{W}_{n} x_{n}\right)-A \mathbb{W}_{n} u_{n}\right\| \\
& +\beta_{n}\left\|x_{n}-\mathbb{W}_{n} u_{n}\right\| .
\end{aligned}
$$

Hence,

$$
\left(1-\delta_{n}\right)\left\|x_{n}-\mathbb{W}_{n} u_{n}\right\| \leq\left\|x_{n+1}-x_{n}\right\|+\mu_{n}\left\|\theta g\left(\mathbb{W}_{n} x_{n}\right)-A \mathbb{W}_{n} u_{n}\right\|
$$

Using the given conditions and (3.21) in (3.22), we get

$$
\lim _{n \rightarrow \infty}\left\|x_{n}-\mathbb{W}_{n} u_{n}\right\|=0
$$

By (3.8) and (3.11), we compute

$$
\begin{aligned}
\left\|x_{n+1}-\tilde{x}\right\|^{2}= & \left\|\mu_{n}\left(\theta g\left(\mathbb{W}_{n} x_{n}\right)-A \tilde{x}\right)+\delta_{n}\left(x_{n}-\mathbb{W}_{n} u_{n}\right)+\left(1-\mu_{n} A\right)\left(\mathbb{W}_{n} u_{n}-\tilde{x}\right)\right\|^{2} \\
\leq & \left\|\left(1-\mu_{n} A\right)\left(\mathbb{W}_{n} u_{n}-\tilde{x}\right)+\delta_{n}\left(x_{n}-\mathbb{W}_{n} u_{n}\right)\right\|^{2} \\
& +2\left\langle\mu_{n} \theta g\left(\mathbb{W}_{n} x_{n}\right)-A \tilde{x}, x_{n+1}-\tilde{x}\right\rangle \\
\leq & {\left[\left\|\left(1-\mu_{n} A\right)\left(\mathbb{W}_{n} u_{n}-\tilde{x}\right)\right\|+\delta_{n}\left\|x_{n}-\mathbb{W}_{n} u_{n}\right\|\right]^{2} } \\
& +2 \mu_{n}\left\langle\theta g\left(\mathbb{W}_{n} x_{n}\right)-A \tilde{x}, x_{n+1}-\tilde{x}\right\rangle \\
\leq & {\left[\left(1-\mu_{n} \bar{\theta}\right)\left\|u_{n}-\tilde{x}\right\|+\delta_{n}\left\|x_{n}-\mathbb{W}_{n} u_{n}\right\|\right]^{2} } \\
& +2 \mu_{n}\left\langle\theta g\left(\mathbb{W}_{n} x_{n}\right)-A \tilde{x}, x_{n+1}-\tilde{x}\right\rangle \\
= & \left(1-\mu_{n} \bar{\theta}\right)^{2}\left\|u_{n}-\tilde{x}\right\|^{2}+\delta_{n}^{2}\left\|x_{n}-\mathbb{W}_{n} u_{n}\right\|^{2} \\
& +2\left(1-\mu_{n} \bar{\theta}\right) \delta_{n}\left\|u_{n}-\tilde{x}\right\|\left\|x_{n}-\mathbb{W}_{n} u_{n}\right\| \\
& +2 \mu_{n}\left\langle\theta g\left(\mathbb{W}_{n} x_{n}\right)-A \tilde{x}, x_{n+1}-\tilde{x}\right\rangle \\
\leq & \left(1-\mu_{n} \bar{\theta}\right)^{2}\left[\left\|x_{n}-\tilde{x}\right\|^{2}+\eta(\epsilon \eta-1)\left\|(Q-I) B x_{n}\right\|^{2}\right] \\
& +\delta_{n}^{2}\left\|x_{n}-\mathbb{W}_{n} u_{n}\right\|^{2}+2\left(1-\mu_{n} \bar{\theta}\right) \delta_{n}\left\|u_{n}-\tilde{x}\right\|\left\|x_{n}-\mathbb{W}_{n} u_{n}\right\| \\
& +2 \mu_{n}\left\langle\theta g\left(\mathbb{W}_{n} x_{n}\right)-A \tilde{x}, x_{n+1}-\tilde{x}\right\rangle \\
= & \left(1-2 \mu_{n} \bar{\theta}+\left(\mu_{n} \bar{\theta}\right)^{2}\right)\left\|x_{n}-\tilde{x}\right\|^{2}+\left(1-\mu_{n} \bar{\theta}\right)^{2} \eta(\epsilon \eta-1)\left\|(Q-I) B x_{n}\right\|^{2}
\end{aligned}
$$




$$
\begin{aligned}
& +\delta_{n}^{2}\left\|x_{n}-\mathbb{W}_{n} u_{n}\right\|^{2}+2\left(1-\mu_{n} \bar{\theta}\right) \delta_{n}\left\|u_{n}-\tilde{x}\right\|\left\|x_{n}-\mathbb{W}_{n} u_{n}\right\| \\
& +2 \mu_{n}\left(\theta g\left(\mathbb{W}_{n} x_{n}\right)-A \tilde{x}, x_{n+1}-\tilde{x}\right\rangle \\
\leq & \left.\left\|x_{n}-\tilde{x}\right\|^{2}+\left(\mu_{n} \bar{\theta}\right)^{2}\right)\left\|x_{n}-\tilde{x}\right\|^{2}+\left(1-\mu_{n} \bar{\theta}\right)^{2} \eta(\epsilon \eta-1)\left\|(Q-I) B x_{n}\right\|^{2} \\
& +\delta_{n}^{2}\left\|x_{n}-\mathbb{W}_{n} u_{n}\right\|^{2}+2\left(1-\mu_{n} \bar{\theta}\right) \delta_{n}\left\|u_{n}-\tilde{x}\right\|\left\|x_{n}-\mathbb{W}_{n} u_{n}\right\| \\
& +2 \mu_{n}\left(\theta g\left(\mathbb{W}_{n} x_{n}\right)-A \tilde{x}, x_{n+1}-\tilde{x}\right) .
\end{aligned}
$$

Therefore,

$$
\begin{aligned}
\left(1-\mu_{n} \bar{\theta}\right)^{2} \eta(1-\epsilon \eta)\left\|(Q-I) B x_{n}\right\|^{2} \leq & \left\|x_{n}-\tilde{x}\right\|^{2}-\left\|x_{n+1}-\tilde{x}\right\|^{2} \\
& +\delta_{n}^{2}\left\|x_{n}-\mathbb{W}_{n} u_{n}\right\|^{2}+\mu_{n} \bar{\theta}^{2}\left\|x_{n}-\tilde{x}\right\|^{2} \\
& +2\left(1-\mu_{n} \bar{\theta}\right) \delta_{n}\left\|u_{n}-\tilde{x}\right\|\left\|x_{n}-\mathbb{W}_{n} u_{n}\right\| \\
& +2 \mu_{n}\left(\theta g\left(\mathbb{W}_{n} x_{n}\right)-A \tilde{x}, x_{n+1}-\tilde{x}\right\rangle \\
\leq & \left(\left\|x_{n}-\tilde{x}\right\|+\left\|x_{n+1}-\tilde{x}\right\|\right)\left\|x_{n}-x_{n+1}\right\| \\
& +\delta_{n}^{2}\left\|x_{n}-\mathbb{W}_{n} u_{n}\right\|^{2}+\mu_{n} \bar{\theta}^{2}\left\|x_{n}-\tilde{x}\right\|^{2} \\
& +2\left(1-\mu_{n} \bar{\theta}\right) \delta_{n}\left\|u_{n}-\tilde{x}\right\|\left\|x_{n}-\mathbb{W}_{n} u_{n}\right\| \\
& +2 \mu_{n}\left(\theta\left\|g\left(\mathbb{W}_{n} x_{n}\right)\right\|\right. \\
& +\|A \tilde{x}\|)\left\|x_{n+1}-\tilde{x}\right\| .
\end{aligned}
$$

Since $\eta(1-\epsilon \eta)>0, \lim _{n \rightarrow \infty} \mu_{n}=0$ and $\left\{x_{n}\right\},\left\{u_{n}\right\}$ are bounded, and using (3.21) and (3.23), we have

$$
\lim _{n \rightarrow \infty}\left\|(Q-I) B x_{n}\right\|^{2}=0
$$

Next, we calculate

$$
\begin{aligned}
\left\|v_{n}-\tilde{x}\right\|^{2}= & \left\|J_{\rho_{1}}^{M_{1}}\left(x_{n}+\eta B^{*}(Q-I) B x_{n}\right)-\tilde{x}\right\|^{2} \\
\leq & \| J_{\rho_{1}}^{M_{1}}\left(x_{n}+\eta B^{*}(Q-I) B x_{n}\right)-J_{\rho_{1}}^{M_{1} \tilde{x} \|^{2}} \\
\leq & \left\langle v_{n}-\tilde{x}, x_{n}+\eta B^{*}(Q-I) B x_{n}-\tilde{x}\right\rangle \\
= & \frac{1}{2}\left\{\left\|v_{n}-\tilde{x}\right\|^{2}+\left\|x_{n}+\eta B^{*}(Q-I) B x_{n}-\tilde{x}\right\|^{2}-\|\left(v_{n}-\tilde{x}\right)\right. \\
& \left.-\left[x_{n}+\eta B^{*}(Q-I) B x_{n}-\tilde{x}\right] \|^{2}\right\} \\
= & \frac{1}{2}\left\{\left\|v_{n}-\tilde{x}\right\|^{2}+\left\|x_{n}-\tilde{x}\right\|^{2}-\left\|v_{n}-x_{n}-\eta B^{*}(Q-I) B x_{n}\right\|^{2}\right\} \\
= & \frac{1}{2}\left\{\left\|v_{n}-\tilde{x}\right\|^{2}+\left\|x_{n}-\tilde{x}\right\|^{2}-\left[\left\|v_{n}-x_{n}\right\|^{2}+\eta^{2}\left\|B^{*}(Q-I) B x_{n}\right\|^{2}\right.\right. \\
& \left.\left.-2 \eta\left\langle v_{n}-x_{n}, B^{*}(Q-I) B x_{n}\right\rangle\right]\right\} .
\end{aligned}
$$

Hence, we obtain

$$
\left\|v_{n}-\tilde{x}\right\|^{2} \leq\left\|x_{n}-\tilde{x}\right\|^{2}-\left\|v_{n}-x_{n}\right\|^{2}+2 \eta\left\|B\left(v_{n}-x_{n}\right)\right\|\left\|(Q-I) B x_{n}\right\| .
$$


By (3.11) and (3.24), we obtain

$$
\begin{aligned}
\left\|x_{n+1}-\tilde{x}\right\|^{2} \leq & \left(1-\mu_{n} \bar{\theta}\right)^{2}\left\|u_{n}-\tilde{x}\right\|^{2}+\delta_{n}^{2}\left\|x_{n}-\mathbb{W}_{n} u_{n}\right\|^{2} \\
& +2\left(1-\mu_{n} \bar{\theta}\right) \delta_{n}\left\|u_{n}-\tilde{x}\right\|\left\|x_{n}-\mathbb{W}_{n} u_{n}\right\| \\
& +2 \mu_{n}\left(\theta g\left(\mathbb{W}_{n} x_{n}\right)-A \tilde{x}, x_{n+1}-\tilde{x}\right\rangle \\
\leq & \left(1-\mu_{n} \bar{\theta}\right)^{2}\left[\left\|x_{n}-\tilde{x}\right\|^{2}-\left\|v_{n}-x_{n}\right\|^{2}\right. \\
& \left.+2 \eta\left\|A\left(u_{n}-x_{n}\right)\right\|\left\|(Q-I) B x_{n}\right\|\right] \\
& +\delta_{n}^{2}\left\|x_{n}-\mathbb{W}_{n} u_{n}\right\|^{2}+2\left(1-\mu_{n} \bar{\theta}\right) \delta_{n}\left\|u_{n}-\tilde{x}\right\|\left\|x_{n}-\mathbb{W}_{n} u_{n}\right\| \\
& +2 \mu_{n}\left\langle\theta g\left(\mathbb{W}_{n} x_{n}\right)-A \tilde{x}, x_{n+1}-\tilde{x}\right\rangle \\
\leq & \left\|x_{n}-\tilde{x}\right\|^{2}+\left(\mu_{n} \bar{\theta}\right)^{2}\left\|x_{n}-\tilde{x}\right\|^{2} \\
& -2 \mu_{n} \bar{\theta}\left\|x_{n}-\tilde{x}\right\|^{2}-\left(1-\mu_{n} \bar{\theta}\right)^{2}\left\|v_{n}-x_{n}\right\|^{2} \\
& +2\left(1-\mu_{n} \bar{\theta}\right)^{2} \eta\left\|A\left(u_{n}-x_{n}\right)\right\|\left\|(Q-I) B x_{n}\right\|+\delta_{n}^{2}\left\|x_{n}-\mathbb{W}_{n} u_{n}\right\|^{2} \\
& +2\left(1-\mu_{n} \bar{\theta}\right) \delta_{n}\left\|u_{n}-\tilde{x}\right\|\left\|x_{n}-\mathbb{W}_{n} u_{n}\right\| \\
& +2 \mu_{n}\left(\theta g\left(\mathbb{W}_{n} x_{n}\right)-A \tilde{x}, x_{n+1}-\tilde{x}\right) .
\end{aligned}
$$

Hence,

$$
\begin{aligned}
\left(1-\mu_{n} \bar{\theta}\right)^{2}\left\|v_{n}-x_{n}\right\|^{2} \leq & \left\|x_{n}-\tilde{x}\right\|^{2}-\left\|x_{n+1}-\tilde{x}\right\|^{2} \\
& +\left(\mu_{n} \bar{\theta}\right)^{2}\left\|x_{n}-\tilde{x}\right\|^{2}-2 \mu_{n} \bar{\theta}\left\|x_{n}-\tilde{x}\right\|^{2} \\
& +2\left(1-\mu_{n} \bar{\theta}\right)^{2} \eta\left\|A\left(u_{n}-x_{n}\right)\right\|\left\|(Q-I) B x_{n}\right\| \\
& +\delta_{n}^{2}\left\|x_{n}-\mathbb{W}_{n} u_{n}\right\|^{2} \\
& +2\left(1-\mu_{n} \bar{\theta}\right) \delta_{n}\left\|u_{n}-\tilde{x}\right\|\left\|x_{n}-\mathbb{W}_{n} u_{n}\right\| \\
& +2 \mu_{n}\left(\theta g\left(\mathbb{W}_{n} x_{n}\right)-A \tilde{x}, x_{n+1}-\tilde{x}\right\rangle \\
\leq & \left(\left\|x_{n}-\tilde{x}\right\|+\left\|x_{n+1}-\tilde{x}\right\|\right)\left\|x_{n}-x_{n+1}\right\| \\
& +\left(\mu_{n} \bar{\theta}\right)^{2}\left\|x_{n}-\tilde{x}\right\|^{2}-2 \mu_{n} \bar{\theta}\left\|x_{n}-\tilde{x}\right\|^{2} \\
& +2\left(1-\mu_{n} \bar{\theta}\right)^{2} \eta\left\|A\left(u_{n}-x_{n}\right)\right\|\left\|(Q-I) B x_{n}\right\| \\
& +\delta_{n}^{2}\left\|x_{n}-\mathbb{\mathbb { W } _ { n }} u_{n}\right\|^{2} \\
& +2\left(1-\mu_{n} \bar{\theta}\right) \delta_{n}\left\|u_{n}-\tilde{x}\right\|\left\|x_{n}-\mathbb{W}_{n} u_{n}\right\| \\
& +2 \mu_{n}\left(\theta\left\|g\left(\mathbb{W}_{n} x_{n}\right)\right\|+\|A \tilde{x}\|\right)\left\|x_{n+1}-\tilde{x}\right\| .
\end{aligned}
$$

As $\left\{x_{n}\right\},\left\{u_{n}\right\}$ are bounded, and using (3.21), (3.23), (3.26) and the given conditions, we have

$$
\lim _{n \rightarrow \infty}\left\|v_{n}-x_{n}\right\|=0
$$

Next, we prove that $\lim _{n \rightarrow \infty}\left\|v_{n}-u_{n}\right\|=0$. 
We estimate

$$
\begin{aligned}
\left\|x_{n+1}-\tilde{x}\right\|^{2} & =\left\|\mu_{n} \theta g\left(\mathbb{W}_{n} x_{n}\right)+\delta_{n} x_{n}+\left(\left(1-\delta_{n}\right) I-\mu_{n} A\right) \mathbb{W}_{n} u_{n}-\tilde{x}\right\|^{2} \\
& =\left\|\left(1-\delta_{n}\right)\left(\mathbb{W}_{n} u_{n}-\tilde{x}\right)+\delta_{n}\left(x_{n}-\tilde{x}\right)+\mu_{n}\left(\theta g\left(\mathbb{W}_{n} x_{n}\right)-A \mathbb{W}_{n} u_{n}\right)\right\|^{2} \\
& \leq\left(1-\delta_{n}\right)\left\|\mathbb{W}_{n} u_{n}-\tilde{x}\right\|^{2}+\delta_{n}\left\|x_{n}-\tilde{x}\right\|^{2}+2 \mu_{n}\left\langle\kappa_{n}, x_{n+1}-\tilde{x}\right\rangle \\
& \leq\left(1-\delta_{n}\right)\left\|\mathbb{W}_{n} u_{n}-\tilde{x}\right\|^{2}+\delta_{n}\left\|x_{n}-\tilde{x}\right\|^{2}+2 \omega^{2} \mu_{n} \\
& \leq\left(1-\delta_{n}\right)\left\|u_{n}-\tilde{x}\right\|^{2}+\delta_{n}\left\|x_{n}-\tilde{x}\right\|^{2}+2 \omega^{2} \mu_{n} .
\end{aligned}
$$

In the above inequality we set $\kappa_{n}=\theta g\left(\mathbb{W}_{n} x_{n}\right)-A \mathbb{W}_{n} u_{n}$, and let $\omega>0$ be a suitable constant with $\omega \geq \sup _{n}\left\{\left\|\kappa_{n}\right\|,\left\|x_{n}-\tilde{x}\right\|\right\}$. Thus,

$$
\begin{aligned}
\left\|x_{n+1}-\tilde{x}\right\|^{2} \leq & \left(1-\delta_{n}\right)\left\|u_{n}-\tilde{x}\right\|^{2}+\delta_{n}\left\|x_{n}-\tilde{x}\right\|^{2}+2 \omega^{2} \mu_{n} \\
\leq & \left(1-\delta_{n}\right)\left\{\left\|P_{C_{1}}\left(v_{n}-\sigma_{n} D v_{n}\right)-P_{C_{1}}\left(\tilde{x}-\sigma_{n} D \tilde{x}\right)\right\|^{2}\right\} \\
& +\delta_{n}\left\|x_{n}-\tilde{x}\right\|^{2}+2 \omega^{2} \mu_{n} \\
\leq & \left(1-\delta_{n}\right)\left\{\left\|v_{n}-\tilde{x}\right\|^{2}+\sigma_{n}\left(\sigma_{n}-2 \gamma\right)\left\|D v_{n}-D \tilde{x}\right\|^{2}\right\} \\
& +\delta_{n}\left\|x_{n}-\tilde{x}\right\|^{2}+2 \omega^{2} \mu_{n} \\
\leq & \left(1-\delta_{n}\right)\left\{\left\|x_{n}-\tilde{x}\right\|^{2}+\sigma_{n}\left(\sigma_{n}-2 \gamma\right)\left\|D v_{n}-D \tilde{x}\right\|^{2}\right\} \\
& +\delta_{n}\left\|x_{n}-\tilde{x}\right\|^{2}+2 \omega^{2} \mu_{n} \\
\leq & \left(1-\delta_{n}\right) \sigma_{n}\left(\sigma_{n}-2 \omega\right)\left\|D v_{n}-D \tilde{x}\right\|^{2} \\
& +\left\|x_{n}-\tilde{x}\right\|^{2}+2 \omega^{2} \mu_{n},
\end{aligned}
$$

which implies

$$
\begin{aligned}
\left(1-\delta_{n}\right) \sigma_{n}\left(2 \omega-\sigma_{n}\right)\left\|D v_{n}-D \tilde{x}\right\|^{2} & \leq\left\|x_{n}-\tilde{x}\right\|^{2}-\left\|x_{n+1}-\tilde{x}\right\|^{2}+2 \omega^{2} \mu_{n} \\
& \leq\left(\left\|x_{n}-\tilde{x}\right\|+\left\|x_{n+1}-\tilde{x}\right\|\right)\left\|x_{n}-x_{n+1}\right\|+2 \omega^{2} \mu_{n} .
\end{aligned}
$$

By (3.21) and the given conditions, we get

$$
\lim _{n \rightarrow \infty}\left\|D v_{n}-D \tilde{x}\right\|=0
$$

From (2.7), we compute

$$
\begin{aligned}
\left\|u_{n}-\tilde{x}\right\|^{2}= & \left\|P_{C_{1}}\left(v_{n}-\sigma_{n} D v_{n}\right)-P_{C_{1}}\left(\tilde{x}-\sigma_{n} D \tilde{x}\right)\right\|^{2} \\
\leq & \left\langle u_{n}-\tilde{x},\left(v_{n}-\sigma_{n} D v_{n}\right)-\left(\tilde{x}-\sigma_{n} D \tilde{x}\right)\right\rangle \\
\leq & \frac{1}{2}\left\{\left\|u_{n}-\tilde{x}\right\|^{2}+\|\left(v_{n}-\sigma_{n} D v_{n}\right)\right. \\
& \left.-\left(\tilde{x}-\sigma_{n} D \tilde{x}\right)\left\|^{2}-\right\|\left(u_{n}-v_{n}\right)+\sigma_{n}\left(D v_{n}-D \tilde{x}\right) \|^{2}\right\} \\
\leq & \frac{1}{2}\left\{\left\|u_{n}-\tilde{x}\right\|^{2}+\left\|v_{n}-\tilde{x}\right\|^{2}-\left\|\left(u_{n}-v_{n}\right)+\sigma_{n}\left(D v_{n}-D \tilde{x}\right)\right\|^{2}\right\} \\
\leq & \left\|v_{n}-\tilde{x}\right\|^{2}-\left\|u_{n}-v_{n}\right\|^{2}-\sigma_{n}^{2}\left\|D v_{n}-D \tilde{x}\right\|^{2}
\end{aligned}
$$




$$
\begin{aligned}
& +2 \sigma_{n}\left\langle u_{n}-v_{n}, D u_{n}-D \tilde{x}\right\rangle \\
\leq & \left\|v_{n}-\tilde{x}\right\|^{2}-\left\|u_{n}-v_{n}\right\|^{2}+2 \sigma_{n}\left\|u_{n}-v_{n}\right\|\left\|D v_{n}-D \tilde{x}\right\| \\
\leq & \left\|x_{n}-\tilde{x}\right\|^{2}-\left\|u_{n}-v_{n}\right\|^{2}+2 \sigma_{n}\left\|u_{n}-v_{n}\right\|\left\|D v_{n}-D \tilde{x}\right\| .
\end{aligned}
$$

By (3.32), we obtain

$$
\begin{aligned}
\left\|x_{n+1}-\tilde{x}\right\|^{2} \leq & \left(1-\delta_{n}\right)\left\|u_{n}-\tilde{x}\right\|^{2}+\delta_{n}\left\|x_{n}-\tilde{x}\right\|^{2}+2 \omega^{2} \mu_{n} \\
\leq & \left(1-\delta_{n}\right)\left\{\left\|x_{n}-\tilde{x}\right\|^{2}-\left\|u_{n}-v_{n}\right\|^{2}\right. \\
& \left.+2 \sigma_{n}\left\|u_{n}-v_{n}\right\|\left\|D v_{n}-D \tilde{x}\right\|\right\}+\delta_{n}\left\|x_{n}-\tilde{x}\right\|^{2}+2 \omega^{2} \mu_{n}
\end{aligned}
$$

which implies

$$
\begin{aligned}
\left(1-\delta_{n}\right)\left\|u_{n}-v_{n}\right\|^{2} \leq & \left\|x_{n}-\tilde{x}\right\|^{2}-\left\|x_{n+1}-\tilde{x}\right\|^{2} \\
& +2\left(1-\delta_{n}\right) \sigma_{n}\left\|u_{n}-v_{n}\right\|\left\|D v_{n}-D \tilde{x}\right\|+2 \omega^{2} \mu_{n} \\
\leq & \left(\left\|x_{n}-\tilde{x}\right\|+\left\|x_{n+1}-\tilde{x}\right\|\right)\left\|x_{n}-x_{n+1}\right\| \\
& +2\left(1-\delta_{n}\right) \sigma_{n}\left\|u_{n}-v_{n}\right\|\left\|D v_{n}-D \tilde{x}\right\|+2 \omega^{2} \mu_{n} .
\end{aligned}
$$

Using (3.21), (3.33) and the given conditions, we get

$$
\lim _{n \rightarrow \infty}\left\|u_{n}-v_{n}\right\|=0
$$

By (3.23), (3.30), and (3.34), we get

$$
\lim _{n \rightarrow \infty}\left\|\mathbb{W}_{n} u_{n}-u_{n}\right\|=0
$$

By Lemma 2.11, we have $\lim _{n \rightarrow \infty}\left\|\mathbb{W} u_{n}-\mathbb{W}_{n} u_{n}\right\|=0$. Thus,

$$
\lim _{n \rightarrow \infty}\left\|\mathbb{W} u_{n}-u_{n}\right\|=0
$$

Step 3. We claim that $\tilde{x} \in \Gamma$.

Since $\left\{x_{n}\right\}$ is bounded, therefore consider $\tilde{x} \in H_{1}$ to be any weak cluster point of $\left\{x_{n}\right\}$. Hence, there exists a subsequence $\left\{x_{n_{j}}\right\}$ of $\left\{x_{n}\right\}$ with $x_{n}-\tilde{x}$. By Lemma 2.7 and (3.35), we have $\tilde{x} \in \bigcap_{i=1}^{\infty} \operatorname{Fix}\left(S_{i}\right)$. And $v_{n_{j}}=J_{\rho_{1}}^{M_{1}}\left[x_{n_{j}}+\eta B^{*}(Q-I) B x_{n_{j}}\right]$ can be rewritten as

$$
\frac{\left(x_{n_{j}}-v_{n_{j}}\right)+B^{*}(Q-I) B x_{n_{j}}}{\rho_{1}} \in M_{1} v_{n_{j}} .
$$

Taking $j \rightarrow \infty$ in (3.37) and by (3.26), (3.30) and the concept of the graph of a maximal monotone mapping, we get $0 \in M_{1} \tilde{x}$, that is, $\tilde{x} \in \operatorname{Sol}(\operatorname{MVIP}(1.6))$. Furthermore, since $\left\{x_{n}\right\}$ and $\left\{v_{n}\right\}$ have the same asymptotical behavior, $B x_{n_{j}} \rightarrow B \tilde{x}$. As $Q$ is nonexpansive, by (3.26)and Lemma 2.7, we get $(I-Q) B \tilde{x}=0$. Hence, by Lemma 2.3, $0 \in f(B \tilde{x})+M_{1} B \tilde{x}$, that is, $B \tilde{x} \in \operatorname{Sol}(\operatorname{MVIP}(1.7))$. Thus, $\tilde{x} \in \Lambda$. 
Next, we prove $\tilde{x} \in \operatorname{Sol}(\operatorname{VIP}(1.1))$. Since $\lim _{n \rightarrow \infty}\left\|v_{n}-u_{n}\right\|=0$ and $\lim _{n \rightarrow \infty}\left\|v_{n}-x_{n}\right\|=0$, there exist subsequences $\left\{v_{n_{i}}\right\}$ and $\left\{u_{n_{i}}\right\}$ of $\left\{v_{n}\right\}$ and $\left\{u_{n}\right\}$, respectively, such that $v_{n_{i}} \rightarrow \tilde{x}$ and $u_{n_{i}} \rightarrow \tilde{x}$.

Define the mapping $\mathbb{M}$ as

$$
\mathbb{M}\left(z_{1}\right)= \begin{cases}D\left(z_{1}\right)+\mathbb{N}_{C_{1}}\left(z_{1}\right), & \text { if } z_{1} \in C_{1}, \\ \emptyset, & \text { if } z_{1} \notin C_{1},\end{cases}
$$

where $\mathbb{N}_{C_{1}}\left(z_{1}\right):=\left\{z_{2} \in H_{1}:\left\langle z_{1}-y, z_{2}\right\rangle \geq 0, \forall y \in C_{1}\right\}$ is the normal cone to $C_{1}$ at $z_{1} \in$ $H_{1}$. Thus, $\mathbb{M}$ is a maximal monotone mapping, and hence $0 \in \mathbb{M} z_{1}$ if and only if $z_{1} \in$ $\operatorname{Sol}(\operatorname{VIP}(1.1))$. Let $\left(z_{1}, z_{2}\right) \in \operatorname{graph}(\mathbb{M})$. Then we have $z_{2} \in \mathbb{M} z_{1}=D z_{1}+\mathbb{N}_{C_{1}}\left(z_{1}\right)$, and hence $z_{2}-D z_{1} \in \mathbb{N}_{C_{1}}\left(z_{1}\right)$. So, we have $\left\langle z_{1}-y, z_{2}-D z_{1}\right\rangle \geq 0$ for all $y \in C_{1}$. On the other hand, from $u_{n}=P_{C_{1}}\left(v_{n}-\sigma_{n} D v_{n}\right)$ and $z_{1} \in C_{1}$, we have

$$
\left\langle\left(v_{n}-\sigma_{n} D v_{n}\right)-u_{n}, u_{n}-z_{1}\right\rangle \geq 0 \text {. }
$$

This implies that

$$
\left\langle z_{1}-u_{n}, \frac{u_{n}-v_{n}}{\sigma_{n}}+D v_{n}\right\rangle \geq 0
$$

Since $\left\langle z_{1}-y, z_{2}-D z_{1}\right\rangle \geq 0$, for all $y \in C_{1}$ and $u_{n_{i}} \in C_{1}$, using the monotonicity of $D$, we have

$$
\begin{aligned}
\left\langle z_{1}-u_{n_{i}}, z_{2}\right\rangle \geq & \left\langle z_{1}-u_{n_{i}}, D z_{1}\right\rangle \\
\geq & \left\langle z_{1}-u_{n_{i}}, D z_{1}\right\rangle-\left\langle z_{1}-u_{n_{i}}, \frac{u_{n_{i}}-v_{n_{i}}}{\sigma_{n}}+D u_{n_{i}}\right\rangle \\
= & \left\langle z_{1}-u_{n_{i}}, D z_{1}-D u_{n_{i}}\right\rangle+\left\langle z_{1}-u_{n_{i}}, D u_{n_{i}}-D v_{n_{i}}\right\rangle \\
& -\left\langle z_{1}-u_{n_{i}}, \frac{u_{n_{i}}-v_{n_{i}}}{\sigma_{n}}\right\rangle \\
\geq & \left\langle z_{1}-u_{n_{i}}, D u_{n_{i}}-D v_{n_{i}}\right\rangle-\left\langle z_{1}-u_{n_{i}}, \frac{u_{n_{i}}-v_{n_{i}}}{\sigma_{n}}\right\rangle .
\end{aligned}
$$

Since $D$ is continuous, on taking limit $i \rightarrow \infty$, we have $\left\langle z_{1}-\tilde{x}, z_{2}\right\rangle \geq 0$. Since $\mathbb{M}$ is maximal monotone, we have $\tilde{x} \in \mathbb{M}^{-1}(0)$ and hence $\tilde{x} \in \operatorname{Sol}(\operatorname{VIP}(1.1))$. Thus, $\tilde{x} \in \Gamma$.

Step 4. Finally, we prove that $\limsup _{n \rightarrow \infty}\left\langle(\theta g-A) z, x_{n}-z\right\rangle \leq 0$, where $z=P_{\Gamma}(I-A+\theta g) z$ and $x_{n} \rightarrow \tilde{x}$.

By (2.3) and (3.23), we obtain

$$
\begin{aligned}
\lim \sup _{n \rightarrow \infty}\left\langle(\theta g-A) z, x_{n}-z\right\rangle & =\lim \sup _{n \rightarrow \infty}\left\langle(\theta g-A) z, \mathbb{W}_{n} u_{n}-z\right\rangle \\
& \leq \lim \sup _{i \rightarrow \infty}\left\langle(\theta g-A) z, \mathbb{W}_{n} u_{n_{i}}-z\right\rangle \\
& =\langle(\theta g-A) z, \tilde{x}-z\rangle \\
& \leq 0 .
\end{aligned}
$$


Using (3.9) and (3.11), we calculate

$$
\begin{aligned}
\left\|x_{n+1}-\tilde{x}\right\|^{2}= & \left\langle\mu_{n}\left(\theta g\left(\mathbb{W}_{n} x_{n}\right)-A \tilde{x}\right)\right. \\
& \left.+\delta_{n}\left(x_{n}-\tilde{x}\right)+\left(\left(1-\delta_{n}\right) I-\mu_{n} A\right)\left(\mathbb{W}_{n} u_{n}-\tilde{x}\right), x_{n+1}-\tilde{x}\right\rangle \\
= & \mu_{n}\left\langle\theta g\left(\mathbb{W}_{n} x_{n}\right)-A \tilde{x}, x_{n+1}-\tilde{x}\right\rangle+\delta_{n}\left\langle x_{n}-\tilde{x}, x_{n+1}-\tilde{x}\right\rangle \\
& +\left\langle\left(\left(1-\delta_{n}\right) I-\mu_{n} A\right)\left(\mathbb{W}_{n} u_{n}-\tilde{x}\right), x_{n+1}-\tilde{x}\right\rangle \\
\leq & \mu_{n}\left(\theta\left\langle g\left(\mathbb{W}_{n} x_{n}\right)-g(\tilde{x}), x_{n+1}-\tilde{x}\right\rangle+\left\langle\theta g(\tilde{x})-A \tilde{x}, x_{n+1}-\tilde{x}\right\rangle\right) \\
& +\delta_{n}\left\|x_{n}-\tilde{x}\right\|\left\|x_{n+1}-\tilde{x}\right\| \\
& +\left\|\left(1-\delta_{n}\right) I-\mu_{n} A\right\|\left\|\mathbb{W} u_{n}-\tilde{x}\right\|\left\|x_{n+1}-\tilde{x}\right\| \\
\leq & \mu_{n} \tau \theta\left\|x_{n}-\tilde{x}\right\|\left\|x_{n+1}-\tilde{x}\right\|+\mu_{n}\left\langle\theta g(\tilde{x})-A \tilde{x}, x_{n+1}-\tilde{x}\right\rangle \\
& +\delta_{n}\left\|x_{n}-\tilde{x}\right\|\left\|x_{n+1}-\tilde{x}\right\|+\left(1-\delta_{n}-\mu_{n} \bar{\theta}\right)\left\|u_{n}-\tilde{x}\right\|\left\|x_{n+1}-\tilde{x}\right\| \\
\leq & \mu_{n} \tau \theta\left\|x_{n}-\tilde{x}\right\|\left\|x_{n+1}-\tilde{x}\right\|+\mu_{n}\left\langle\theta g(\tilde{x})-A \tilde{x}, x_{n+1}-\tilde{x}\right\rangle \\
& +\delta_{n}\left\|x_{n}-\tilde{x}\right\|\left\|x_{n+1}-\tilde{x}\right\|+\left(1-\delta_{n}-\mu_{n} \bar{\theta}\right)\left\|x_{n}-\tilde{x}\right\|\left\|x_{n+1}-\tilde{x}\right\| \\
= & {\left[1-\mu_{n}(\bar{\theta}-\theta \tau)\right]\left\|x_{n}-\tilde{x}\right\|\left\|x_{n+1}-\tilde{x}\right\|+\mu_{n}\left\langle\theta g(\tilde{x})-A \tilde{x}, x_{n+1}-\tilde{x}\right\rangle } \\
& +\mu_{n}\left\langle\theta g(\tilde{x})-A \tilde{x}, x_{n+1}-\tilde{x}\right\rangle, \\
\leq & +\mu_{n}(\bar{\theta}-\theta \tau) \\
& +\mu_{n}\left\langle\theta g(\tilde{x})-A \tilde{x}, x_{n+1}-\tilde{x}\right\rangle \\
\leq & \frac{1-\mu_{n}(\bar{\theta}-\theta \tau)}{2}\left\|x_{n}-\tilde{x}\right\|^{2}+\frac{1}{2}\left\|x_{n+1}-\tilde{x}\right\|^{2} \\
2 & \left.\left\|x_{n+1}-\tilde{x}\right\|^{2}\right) \\
& \\
& \\
&
\end{aligned}
$$

which yields that

$$
\begin{aligned}
\left\|x_{n+1}-\tilde{x}\right\|^{2} \leq & {\left[1-\mu_{n}(\bar{\theta}-\theta \tau)\right]\left\|x_{n}-\tilde{x}\right\|^{2} } \\
& +2 \mu_{n}\left(\left\langle\theta g(\tilde{x})-A \tilde{x}, x_{n+1}-\tilde{x}\right\rangle\right. \\
= & {\left[1-\mu_{n}(\bar{\theta}-\theta \tau)\right]\left\|x_{n}-\tilde{x}\right\|^{2}+2 \mu_{n}\left\langle\theta g(\tilde{x})-A \tilde{x}, x_{n+1}-\tilde{x}\right\rangle . }
\end{aligned}
$$

Thus, by (3.39), (3.41), Lemma 2.6 and using $\lim _{n \rightarrow \infty} \mu_{n}=0$, we get $x_{n} \rightarrow \tilde{x}$, where $\tilde{x}=$ $P_{\Gamma}(I+\theta g-A)$.

Now, we list the following consequences from Theorem 3.1.

Corollary 3.1 Let $H_{1}$ and $H_{2}$ denote the Hilbert spaces and $C_{1} \subset H_{1}$ be a nonempty closed convex subset of Hilbert space $H_{1}$. Let $D: C_{1} \rightarrow H_{1}$ be a $\gamma$-inverse strongly monotone mapping, $B: H_{1} \rightarrow H_{2}$ be a bounded linear operator with its adjoint operator $B^{*}, M_{1}: C_{1} \rightarrow$ $2^{H_{1}}$, and $M_{2}: H_{2} \rightarrow 2^{H_{2}}$ be multi-valued maximal monotone operators and $f: H_{2} \rightarrow H_{2}$ be an $\alpha$-inverse strongly monotone mapping. Let $g: C_{1} \rightarrow C_{1}$ be a contraction mapping with constant $\tau \in(0,1), A$ be a strongly positive bounded linear self-adjoint operator on $C_{1}$ with constant $\bar{\theta}>0$ such that $0<\theta<\frac{\bar{\theta}}{\tau}<\theta+\frac{1}{\tau}$, and $S: C_{1} \rightarrow C_{1}$ be a nonexpansive mapping 
such that $\Gamma:=\Lambda \cap \operatorname{Sol}(\operatorname{VIP}(1.1)) \cap \operatorname{Fix}(S)) \neq \emptyset$. Let $\left\{x_{n}\right\}$ be a sequence generated as follows:

$$
\left.\begin{array}{l}
x_{1} \in C_{1}, \\
v_{n}=J_{\rho_{1}}^{M_{1}}\left[x_{n}+\eta B^{*}(Q-I) B x_{n}\right], \\
u_{n}=P_{C_{1}}\left(v_{n}-\sigma_{n} D v_{n}\right), \\
x_{n+1}=\mu_{n} \theta g\left(S x_{n}\right)+\delta_{n} x_{n}+\left(\left(1-\delta_{n}\right) I-\mu_{n} A\right) S u_{n},
\end{array}\right\}
$$

where $Q=J_{\rho_{2}}^{f, M_{2}}\left(I-\rho_{2} f\right),\left\{\mu_{n}\right\},\left\{\delta_{n}\right\} \subset(0,1)$, and $\eta \in\left(0, \frac{1}{\epsilon}\right), \epsilon$ is the spectral radius of $B^{*} B$. Let the control sequences satisfy the following conditions:

(i) $\lim _{n \rightarrow \infty} \mu_{n}=0, \sum_{n=1}^{\infty} \mu_{n}=\infty$;

(ii) $\rho_{1}>0,0<\rho_{2}<2 \alpha$;

(iii) $0<\liminf _{n \rightarrow \infty} \delta_{n} \leq \limsup _{n \rightarrow \infty} \delta_{n}<1$;

(iv) $0<\liminf _{n \rightarrow \infty} \sigma_{n} \leq \limsup _{n \rightarrow \infty} \sigma_{n}<2 \gamma ; \sum_{n=1}^{\infty}\left|\sigma_{n+1}-\sigma_{n}\right|<\infty$.

Then the sequence $\left\{x_{n}\right\}$ converges strongly to some $\tilde{x} \in \Gamma$, where $\tilde{x}=P_{\Gamma}(\theta g+(I-A)) \tilde{x}$, which solves

$$
\langle(A-\theta g) \tilde{x}, v-\tilde{x}\rangle \geq 0, \quad \forall v \in \Gamma .
$$

Corollary 3.2 Let $H_{1}$ and $H_{2}$ denote the Hilbert spaces and $C_{1} \subset H_{1}$ be a nonempty closed convex subset of Hilbert space $H_{1}$. Let $D: C_{1} \rightarrow H_{1}$ be a $\gamma$-inverse strongly monotone mapping, $B: H_{1} \rightarrow H_{2}$ be a bounded linear operator with its adjoint operator $B^{*}, M_{1}: C_{1} \rightarrow$ $2^{H_{1}}$, and $M_{2}: H_{2} \rightarrow 2^{H_{2}}$ be multi-valued maximal monotone operators. Let $g: C_{1} \rightarrow C_{1}$ be a contraction mapping with constant $\tau \in(0,1)$, A be a strongly positive bounded linear selfadjoint operator on $C_{1}$ with constant $\bar{\theta}>0$ such that $0<\theta<\frac{\bar{\theta}}{\tau}<\theta+\frac{1}{\tau}$, and $S: C_{1} \rightarrow C_{1}$ be a nonexpansive mapping such that $\left.\Gamma:=\operatorname{Sol}\left(\operatorname{S}_{\mathrm{P}} \mathrm{NPP}(1.4)-(1.5)\right) \cap \operatorname{Sol}(\mathrm{VIP}(1.1)) \cap \mathrm{Fix}(S)\right) \neq \emptyset$. Let $\left\{x_{n}\right\}$ be a sequence generated as follows:

$$
\left.\begin{array}{l}
x_{1} \in C_{1}, \\
v_{n}=J_{\rho_{1}}^{M_{1}}\left[x_{n}+\eta B^{*}\left(J_{\rho_{2}}^{M_{2}}-I\right) B x_{n}\right], \\
u_{n}=P_{C_{1}}\left(v_{n}-\sigma_{n} D v_{n}\right), \\
x_{n+1}=\mu_{n} \theta g\left(S x_{n}\right)+\delta_{n} x_{n}+\left(\left(1-\delta_{n}\right) I-\mu_{n} A\right) S u_{n},
\end{array}\right\}
$$

where $\left\{\mu_{n}\right\},\left\{\delta_{n}\right\} \subset(0,1)$ and $\eta \in\left(0, \frac{1}{\epsilon}\right), \epsilon$ is the spectral radius of $B^{*} B$. Let the control sequences satisfy the following conditions:

(i) $\lim _{n \rightarrow \infty} \mu_{n}=0, \sum_{n=1}^{\infty} \mu_{n}=\infty$;

(ii) $0<\liminf _{n \rightarrow \infty} \delta_{n} \leq \limsup _{n \rightarrow \infty} \delta_{n}<1$;

(iii) $0<\liminf _{n \rightarrow \infty} \sigma_{n} \leq \limsup _{n \rightarrow \infty} \sigma_{n}<2 \gamma ; \sum_{n=1}^{\infty}\left|\sigma_{n+1}-\sigma_{n}\right|<\infty$.

Then the sequence $\left\{x_{n}\right\}$ converges strongly to some $\tilde{x} \in \Gamma$, where $\tilde{x}=P_{\Gamma}(\theta g+(I-A)) \tilde{x}$, which solves

$$
\langle(A-\theta g) \tilde{x}, v-\tilde{x}\rangle \geq 0, \quad \forall v \in \Gamma
$$

\section{Numerical example}

Example 4.1 Let $H_{1}=H_{2}=\mathbb{R}$, the set of all real numbers, with the inner product defined by $\langle x, y\rangle=x y, \forall x, y \in \mathbb{R}$, and the induced usual norm $|\cdot|$. Let $C_{1}=[0, \infty)$; let the mapping $f: \mathbb{R} \rightarrow \mathbb{R}$ be defined by $f(y)=y+6, \forall y \in H_{2}$; let $M_{1}: C_{1} \rightarrow 2^{\mathbb{R}}, M_{2}: \mathbb{R} \rightarrow 2^{\mathbb{R}}$ be defined by 
$M_{1}(x)=\{3 x-2\}, \forall x \in C_{1}$ and $M_{2}(y)=\{3 y\}, \forall y \in \mathbb{R}$; let the mapping $B: \mathbb{R} \rightarrow \mathbb{R}$ be defined by $B(x)=-\frac{9}{4} x, \forall x \in \mathbb{R}$; let the mappings $\left\{S_{i}\right\}_{i=1}^{\infty}: C_{1} \rightarrow C_{1}$ be defined by $S_{i} x=\frac{x+2 i}{1+3 i}$ for each $i \in \mathbb{N}$, let the mapping $D: C_{1} \rightarrow \mathbb{R}$ be defined by $D x=3 x-2, \forall x \in C_{1}$; let the mapping $g: C_{1} \rightarrow C_{1}$ be defined by $g(x)=\frac{x}{5}, \forall x \in C_{1}$ and $A x=\frac{x}{2}$ with $\theta=\frac{1}{10}$. Setting $\left\{\mu_{n}\right\}=\left\{\frac{1}{10 n}\right\}$, $\left\{\delta_{n}\right\}=\left\{\frac{1}{2 n^{2}}\right\},\left\{\sigma_{n}\right\}=\frac{1}{4}$, and $\left\{\lambda_{n}\right\}=\left\{\frac{1}{3 n^{2}}\right\}, \forall n \geq 1$. Let $\mathbb{W}_{n}$ be the $\mathbb{W}$-mapping generated by $S_{1}, S_{2}, \ldots$, and $\lambda_{1}, \lambda_{2}, \ldots$ which is defined by (1.10). Then there are sequences $\left\{x_{n}\right\},\left\{u_{n}\right\}$, and $\left\{v_{n}\right\}$ as follows: Given $x_{1}$,

$$
\left.\begin{array}{l}
t_{n}=Q B x_{n}=f_{\rho_{2}}^{f, M_{2}}\left(I-\rho_{2} f\right) B x_{n} \\
y_{n}=x_{n}+\eta B^{*}\left(t_{n}-B x_{n}\right) \\
v_{n}=J_{\rho_{1}}^{M_{1}} y_{n} \\
u_{n}=P_{C_{1}}\left(v_{n}-\sigma_{n} D v_{n}\right), \\
x_{n+1}=\mu_{n} \theta g\left(\mathbb{W}_{n} x_{n}\right)+\delta_{n} x_{n}+\left(\left(1-\delta_{n}\right) I-\mu_{n} A\right) \mathbb{W}_{n} u_{n} .
\end{array}\right\}
$$

Then $\left\{x_{n}\right\}$ converges to $\tilde{x}=\left\{\frac{2}{3}\right\} \in \Gamma$.

Proof Obviously, $B$ is a bounded linear operator on $\mathbb{R}$ with adjoint $B^{*}$ and $\|B\|=\left\|B^{*}\right\|=\frac{9}{4}$, and hence $\eta \in\left(0, \frac{16}{81}\right)$. Therefore, we choose $\eta=0.1$. Further, $f$ is 1 -ism, $\rho_{1}=\frac{1}{4}>0$ and thus $\rho_{2} \subset(0,2)$, so we take $\rho_{2}=\frac{1}{4}$. For each $i, S_{i}$ is nonexpansive with $\operatorname{Fix}\left(S_{i}\right)=\left\{\frac{2}{3}\right\}$. Further, $D$ is 3 -ism and $\operatorname{Sol}(\operatorname{VIP}(1.1))=\left\{\frac{2}{3}\right\}$. Furthermore, $\operatorname{Sol}(\operatorname{MVIP}(1.6))=\left\{\frac{2}{3}\right\}$ and $\operatorname{Sol}(\operatorname{MVIP}(1.7))=$ $\left\{-\frac{3}{2}\right\}$, and thus $\Lambda=\left\{\frac{2}{3} \in C_{1}: \frac{2}{3} \in \operatorname{Sol}(\operatorname{MVIP}(1.6)): B\left(\frac{2}{3}\right) \in \operatorname{Sol}(\operatorname{MVIP}(1.7))\right\}=\left\{\frac{2}{3}\right\}$. Therefore, $\Gamma:=\Lambda \cap \operatorname{Sol}(\operatorname{VIP}(1.1)) \cap\left(\bigcap_{i=1}^{\infty} \operatorname{Fix}\left(S_{i}\right)\right) \neq \emptyset$. Simplify(4.1)as follows: Given $x_{1}$,

$$
\begin{aligned}
& t_{n}=\frac{-27 x_{n}-24}{28} ; \quad y_{n}=\frac{79 x_{n}-36 t_{n}}{160} ; \\
& v_{n}=\frac{4}{7} y_{n}+\frac{2}{7} ; \\
& u_{n}=P_{C_{1}}\left(v_{n}-\sigma_{n} D v_{n}\right) ; \\
&= \begin{cases}0, & \text { if } x<0, \\
1, & \text { if } x>1, \\
\frac{v_{n}+2}{4} & \text { otherwise; }\end{cases} \\
& \mathbb{W}_{n}=x_{n} ;
\end{aligned}
$$

Step 1:

$i=1$;

$\mathbb{W}_{n}=\left(\frac{1}{3 n^{2}}\right) \frac{\left(\mathbb{W}_{n}+2 i\right)}{1+3 i}+\left(1-\frac{1}{3 n^{2}}\right) x_{n} ;$

$i=i+1$

if $(i \leq N)$ go to Step 1 ;

$\mathbb{W}_{n}^{\prime}=u_{n}$

Step $1^{\prime}$ :

$i=1$; 
Figure 1 Convergence of $\left\{x_{n}\right\}$

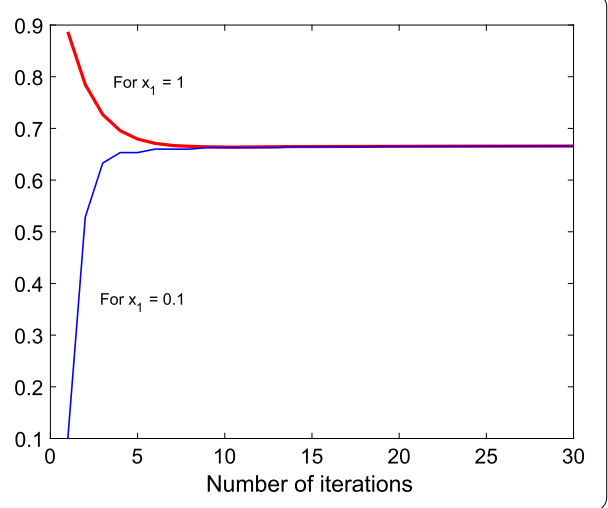

Figure 2 Convergence of $\left\|W_{n} x-W x\right\|$

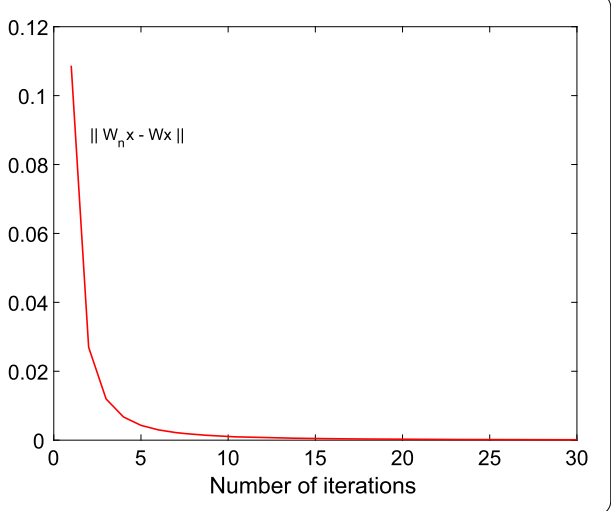

$$
\begin{aligned}
& \mathbb{W}_{n}^{\prime}=\left(\frac{1}{3 n^{2}}\right) \frac{\left(\mathbb{W}_{n}^{\prime}+2 i\right)}{1+3 i}+\left(1-\frac{1}{3 n^{2}}\right) u_{n} ; \\
& i=i+1 ; \\
& \text { if }(i \leq \mathbb{N}) \quad \text { go to Step } 1^{\prime} ; \\
& x_{n+1}=\mu_{n} \theta \frac{\mathbb{W}_{n} x_{n}}{5}+\delta_{n} x_{n}+\left(\left(1-\delta_{n}\right) I-\mu_{n} A\right) \mathbb{W}_{n}^{\prime} u_{n},
\end{aligned}
$$

Finally, by the software Matlab 7.8.0, we obtain the Figures 1 and 2 which show that $\left\{x_{n}\right\}$ converges to $\tilde{x}=\frac{2}{3}$ as $n \rightarrow+\infty$, and $\lim _{n \rightarrow \infty}\left\|\mathbb{W}_{n} x-\mathbb{W} x\right\|=0$ for each $x \in C_{1}$.

\section{Acknowledgements}

This work was supported by the Deanship of Scientific Research (DSR), King Abdulaziz University, Jeddah, under grant No. (D-642-363-1441). The authors, therefore, gratefully acknowledge the DSR technical and financial support.

Funding

This research was funded by King Abdulaziz University, Jeddah, KSA.

\section{Availability of data and materials}

Not applicable.

\section{Competing interests}

The authors declare that they have no competing interests.

\section{Authors' contributions}

All authors contributed equally and significantly in writing this paper. All authors read and approved the final manuscript. 


\section{Author details}

'King Abdulaziz University, Jeddah, Kingdom of Saudi Arabia. ${ }^{2}$ Department of Mathematics, Deanship of Educational Services, Qassim University, Buraidah 51452, Al-Qassim, Saudi Arabia. ${ }^{3}$ Department of Mathematics, Jamia Millia Islamia, 110025 New Delhi, India.

\section{Publisher's Note}

Springer Nature remains neutral with regard to jurisdictional claims in published maps and institutional affiliations.

Received: 25 July 2020 Accepted: 1 September 2020 Published online: 10 September 2020

References

1. Hartman, P., Stampacchia, G.: On some non-linear elliptic differential-functional equation. Acta Math. 115, 271-310 (1966)

2. Moudafi, A.: Split monotone variational inclusions. J. Optim. Theory Appl. 150, 275-283 (2011)

3. Byrne, C.: Iterative oblique projection onto convex sets and the split feasibility problem. Inverse Probl. 18, 441-453 (2002)

4. Censor, Y., Bortfeld, T., Martin, B., Trofimov, A.: A unified approach for inversion problems in intensity modulated radiation therapy. Phys. Med. Biol. 51, $2353-2365$ (2006)

5. Combettes, P.L.: The convex feasibility problem in image recovery. Adv. Imaging Electron Phys. 95, 155-453 (1996)

6. Byrne, C., Censor, Y., Gibali, A., Reich, S.: Weak and strong convergence of algorithms for the split common null point problem. J. Nonlinear Convex Anal. 13(4), 759-775 (2012)

7. Kazmi, K.R., Rizvi, S.H.: An iterative method for split variational inclusion problem and fixed point problem for a nonexpansive mapping. Optim. Lett. 8(3), 1113-1124 (2014)

8. Kazmi, K.R., Ali, R., Furkan, M.: Hybrid iterative method for split monotone variational inclusion problem and hierarchical fixed point problem for a finite family of nonexpansive mappings. Numer. Algorithms 79, 499-527 (2018)

9. Shehu, Y., Ogbuisi, F.U.: An iterative method for solving split monotone variational inclusion and fixed point problems. Rev. R. Acad. Cienc. Exactas Fís. Nat., Ser. A Mat. 110(2), 503-518 (2016)

10. Qin, X., Shang, M., Su, Y.: Strong convergence of a general iterative algorithm for equilibrium problems and variational inequality problems. Math. Comput. Model. 48(7-8), 1033-1046 (2008)

11. Farid, M., Kazmi, K.R.: A new mapping for finding a common solution of split generalized equilibrium problem, variational inequality problem and fixed point problem. Korean J. Math. 27(2), 295-325 (2019)

12. Kangtunyakarn, A., Suantai, S.: A new mapping for finding common solutions of equilibrium problems and fixed point problems of finite family of nonexpansive mappings. Nonlinear Anal. 71(10), 4448-4460 (2009)

13. Opial, Z:: Weak convergence of the sequence of successive approximations for nonexpansive mappings. Bull. Am. Math. Soc. 73(4), 595-597 (1967)

14. Bauschke, H.H., Combettes, P.L.: Convex Analysis and Monotone Operator Theory in Hilbert Spaces. Springer, New York (2011)

15. Suzuki, T.: Strong convergence of Krasnoselskii and Mann's type sequences for one parameter nonexpansive semigroups without Bochner integrals. J. Math. Anal. Appl. 305, 227-239 (2005)

16. Marino, G., Xu, H.K.: A general iterative method for nonexpansive mappings in Hilbert spaces. J. Math. Anal. Appl. 318, 43-52 (2006)

17. Xu, H.K.: Viscosity approximation method for nonexpansive mappings. J. Math. Anal. Appl. 298, 279-291 (2004)

18. Geobel, K., Kirk, W.A.: Topics in Metric Fixed Point Theory. Cambridge Studies in Advanced Mathematics. Cambridge University Press, Cambridge (1990)

19. Shimoji, K., Takahashi, W.: Strong convergence to common fixed points of infinite nonexpansive mappings and applications. Taiwan. J. Math. 5(2), 387-404 (2001)

20. Cho, Y.J., Qin, X., Kang, S.M.: Some results for equilibrium problems and fixed point problems in Hilbert spaces. J. Comput. Anal. Appl. 11(2), 287-294 (2009)

\section{Submit your manuscript to a SpringerOpen ${ }^{\circ}$ journal and benefit from:}

- Convenient online submission

- Rigorous peer review

- Open access: articles freely available online

- High visibility within the field

- Retaining the copyright to your article

Submit your next manuscript at $\gg$ springeropen.com 